\title{
Suppression of vortex-induced vibrations of rigid circular cylinder on springs by localized surface roughness at $3 \times 10^{4} \leq \mathrm{Re} \leq 1.2 \times 10^{5}$
}

Hongrae Park ${ }^{1,4}$, Ph.D., R. Ajith Kumar ${ }^{1,3,4}$, Ph.D., Michael. M. Bernitsas ${ }^{1,2,4,5,6}$, Ph.D.

(1) Department of Mechanical Engineering, University of Michigan, Ann Arbor

(2) Department of Naval Architecture and Marine Engineering, University of Michigan, Ann Arbor

(3) Professor, Department of Mechanical Engineering, AMRITA University, Amritapuri P.O, Kerala State, India

(4) Marine Renewable Energy Laboratory, University of Michigan

(5) CTO, Vortex Hydro Energy, Ann Arbor, Michigan

(6) Corresponding Author: Professor Michael M. Bernitsas, Dept. of NA\&ME, University of Michigan, 2600 Draper Drive, Ann Arbor, Michigan 48109-2145

Also, CTO, Vortex Hydro Energy

Phone: (734) 764-9317, Fax: (734) 936-8820, E-mail: michaelb@umich.edu

Submitted for Publication to:

\section{Ocean Engineering}

Initial submission: $\quad$ October 2014

Revision submission: $\quad$ October 2015 


\begin{abstract}
Suppression of Vortex-Induced Vibrations (VIV) of an elastically mounted circular cylinder in a steady flow is studied experimentally using localized surface roughness, called Passive Turbulence Control (PTC). PTC consists of two roughness strips with thickness on the order of the boundary layer thickness and placed parallel to the cylinder axis symmetrically with respect to the flow. The range of Reynolds number $\left(3 \times 10^{4} \leq \mathrm{Re} \leq 1.2 \times 10^{5}\right)$ considered covers primarily the Transition to Shear Layer3, highlift, flow regime. For the smooth cylinder, the broad synchronization range and higher peak amplitude in the upper branch and unstable oscillations in the lower branch are characteristic of VIV at higher Reynolds numbers and distinctive from those of lower Reynolds numbers. From the PTC-cylinder results with systematic variation of the PTC location, one strong-suppression zone and two weak-suppression zones are identified based on the location of the PTC. These zones are part of the Map that established the relation between PTC and Flow-Induced Motions in previous work. In the strong suppression zone, reduction of $30 \%-80 \%$ is achieved while in the weak suppression zones reduction of less than $30 \%$ is achieved. Broad field-of-view flow visualization shows that smooth cylinder vortex structures change with PTC and between zones.
\end{abstract}

Key words: Vortex-induced vibrations, passive turbulence control, surface roughness, suppression, flow-induced motions, wake vortex structures, circular cylinder

\title{
Nomenclature
}

$A * \quad$ amplitude ratio $A / D$, average of the 60 highest peaks

BDC Bottom Dead Center

c damping coefficient of oscillator

D cylinder diameter

$f_{n, w}$

$=2 \pi \sqrt{\frac{K}{m_{\text {osc }}+m_{A}}}$, natural frequency in still water

$f_{\text {osc }} \quad$ body oscillation frequency

$f^{*} \quad=\frac{f_{\text {osc }}}{f_{n, w}}$ frequency ratio

FIM Flow Induced Motions 


$\begin{array}{ll}H & \text { total thickness of roughness strip } \\ k & \text { average height of grit } \\ K & \text { spring constant } \\ L & \text { cylinder length } \\ m_{A} & =C_{A} \cdot m_{d} \text { ideal added mass with } C_{A}=1 \\ m_{\text {cylinder }} & \text { mass of cylinder } \\ m_{d} & \text { displaced mass of cylinder } \\ m_{\text {osc }} & \text { total oscillating mass including } 1 / 3^{\text {rd }} \text { of the spring mass } \\ m^{*} & =m_{o s c} /\left(\pi \rho D^{2} L / 4\right) \text { mass ratio } \\ p & \text { height of backup paper } \\ \text { PTC } & \text { Passive Turbulence Control } \\ \text { P180: } 60^{\circ}-76^{\circ} \text { two symmetric strips P180 with leading edge at } \pm 60^{\circ} \text { and } 16^{\circ} \text { width } \\ R & \text { vortex radius } \\ \text { Re } & =U D / v \text { Reynolds number } \\ \text { Sc } & =m_{o s c} \zeta / \rho D^{2} \text { Scruton number } \\ \mathrm{t} & \text { time } \\ \text { T } & \text { a period of oscillation } \\ \text { TDC } & \text { Top Dead Center } \\ \text { TrSL3 } & \text { Transition Shear Layer } 3 \\ U & \text { water velocity } \\ U^{*} & =\frac{U}{f_{n, w} D} \text { reduced velocity } \\ \text { VIV } & \text { Vortex Induced Vibrations }\end{array}$

\section{Greek Symbols}

$\alpha \quad$ location of leading edge of PTC in degrees

$\Gamma \quad$ vortex circulation

$\zeta \quad=\frac{c}{2 \sqrt{K m_{\text {osc }}}}$ damping ratio in air

$v \quad$ water kinematic viscosity

$\rho \quad$ water density at $15^{\circ} \mathrm{C}$ 


\section{Introduction}

Flow-Induced Motions (FIM) are frequently encountered Fluid-Structure Interaction (FSI) phenomena when a flexible body is placed in a flow. Among flow-induced motions, the most well-known phenomenon is Vortex-Induced Vibrations (VIV). When an elastically mounted circular cylinder is exposed to a steady transverse flow, shedding vortices apply oscillatory forces on the cylinder resulting in VIV. VIV is characterized by synchronization or "lock-in". In the synchronization range, the vortex shedding frequency and the oscillation frequency of the cylinder are nearly equal and large body motions are observed. For a low mass damping system, the large body motions are more severe than a high mass damping system. Three distinctive response branches are observed; namely, the initial branch, the upper branch, and the lower branch instead of the initial branch, and the lower branch in high mass damping system (Govardhan and Williamson 2000). VIV suppression in a low mass damping system is more challenging in practice due to higher amplitude response. VIV can be seen frequently in a multitude of engineering applications such as heat exchanger tubes, cooling towers, bridges, buildings, offshore structures, and nuclear reactors (Païdoussis 1983; Wakahara et al. 1992). VIV have been studied experimentally and numerically (Bearman 1984; Gabbai and Benaroya 2005; Sarkpaya 1979, 2004; Williamson and Govardhan 2004). Nevertheless, other phenomena such as galloping, flutter, and buffeting are also encountered in practice. Galloping is another form of FIM that non-circular cylinders undergo due to asymmetry or upstream turbulence (Blevins 1990). These FIM adversely affect the safety of structures by shortening their fatigue life (Baarholm et al. 2006). 
Research on developing both passive and active control to attenuate VIV is ongoing (Muddada and Patnaik 2010; Zdravkovich 1981). Only the pertinent literature on passive control is discussed here. Conventional passive control strategies to subdue VIV based on add-on devices are discussed by Blevins (1990) and Kumar et al. (2008). According to Zdravkovich (1981), these devices fall into three categories: (1) Surface protrusions, which affect separation lines and /or separated shear layers. (2) Shrouds, which affect the entrainment layers. (3) Near-wake stabilizers, which affect the switch of the confluence point. Zdravkovich (1981) further introduced classification into (a) omni-directional (flow-direction independent) and (b) uni-directional (flow-direction dependent) devices. Suppression can also be achieved by increasing the system damping or changing its natural frequency (Every et al. 1982). This, however, may increase the overall cost of the structure. Straight fins have been used as suppression devices but may result in amplification of oscillations depending on their location on a cylinder (Gartshore et al. 1978). Effectiveness of a suppression device depends on its ability to suppress vibrations without incurring significant drag penalty. In theory, an effective VIV suppression device could potentially be able to decrease drag rather than increase it (Assi et al. 2009). Helical strakes, perforated shrouds, ribbon cables, spoilers and axial slats can reduce VIV amplitudes by about 70\%-90\% but increase drag (Wong and Kokkalis 1982). On the other hand, streamlined fairings or guide vanes can reduce amplitudes by $80 \%$ or more and result in drag reduction up to 50\% (Blevins 1990). Results by Korkischko and Meneghini (2010) show that helical strakes are very effective in subduing VIV oscillations with amplitude reduction depending on the pitch and height of the strakes. Wong and Kokkalis (1982) compared the suppression performance of perforated shroud, 
helical strakes, and longitudinal slats under identical conditions and found that overall performance is best for slats over a broad range of Scruton number at subcritical Reynolds numbers. Wong and Kokkalis further found that the drag coefficient is the lowest for perforated shroud and the highest for helical strakes. A damage rate for fully straked cylinder, however, was reported to be about eight orders of magnitude lower than that of a bare cylinder (Schaudt et al. 2008). Furthermore, Tombazis and Bearman (1997) researched three-dimensional geometric disturbance effect on bluff body wakes and reduced the base drag using a spanwise wavy trailing edge. Several patents have been issued in the past few decades on suppression devices (Kumar et al. 2008), the majority of them being modifications of existing ones. The more effective the suppression device is, the farther downstream the vortex formation region is located (Zdravkovich 1981). The best suppression devices available at present may bring a maximum amplitude reduction of about $96 \%$ but typically at a high drag-penalty (Lubbad et al. 2007).

Even though there are many studies revealing the effect of surface roughness on flow around a circular cylinder (Achenbach 1971; Achenbach and Heinecke 1981; Güven et al. 1980; Nakamura and Tomonari 1982), roughness has not been perceived as a possible means of suppression in previous studies. Bernitsas and Raghavan $(2008,2011)$ and Chang et al. (2011) studied the effect of localized surface roughness, called passive turbulence control (PTC), on cylinder response. They found that PTC causes substantial changes in the boundary layer characteristics and thereby on the cylinder dynamics. Further, Park et al. (2011, 2013a) researched the cylinder response with respect to the circumferential location of the PTC. This study was carried out experimentally, for a broad range of parameters to design an effective yet simple device, which can act as a 
FIM suppressor as well as an amplifier depending on its method of application. The response of the cylinder was classified with respect to the circumferential location of the PTC. This classification has lead to the construction of the response map of roughnessinduced FIM, hereafter called "PTC-to-FIM Map" for two different roughness configurations (P180 and P60) as shown in Figs. 1(a)-(b), respectively. For both P180 and P60 roughness strips, the maps are similar with nearly identical zones. The identified zones starting from the front stagnation point are weak suppression 1 (WS1), hard galloping 1 (HG1), soft galloping (SG), hard galloping 2 (HG2), strong suppression (SS), and weak suppression 2 (WS2). Even though the overall response classification in the PTC-to-FIM Map is similar for P180 and P60 roughness strips, the surface roughness $k / D$ ( $k=$ average height of grit, $D=$ cylinder diameter) affects slightly the zonal ranges of HG1, SG, HG2, and SS but does not change the order of the zones in the PTC-to-FIM Map. The major objective of this paper is the application of a local roughness strip as a means of suppression in the Reynolds range of $3 \times 10^{4} \leq \mathrm{Re} \leq 1.2 \times 10^{5}$ covering primarily the 'transition to shear layer 3 (TrSL3)', high-lift flow regime (Zdravkovich 1997). For comparison, the smooth cylinder response is also studied in this paper particularly since smooth cylinder studies in the TrSL3 flow regime are rare. The smooth cylinder data in the TrSL3 flow regime presented here are distinctively different from the response of smooth cylinders in lower Reynolds numbers in terms of amplitude, branch, and vortex structures.

The experimental set-up is described in section 2 including the facility, the oscillator, and the Passive Turbulence Control (PTC). The smooth cylinder response and its wake vortex structures are presented in section 3 . Weak suppression and strong suppression 
response are studied in sections 4 and 5, respectively.

\section{Experimental set-up}

All experiments were conducted in the Low Turbulence Free Surface Water (LTFSW) Channel of the Marine Renewable Energy Laboratory (MRELab) at the University of Michigan. The Reynolds number range was $3 \times 10^{4} \leq \mathrm{Re} \leq 1.2 \times 10^{5}$.

The LTFSW Channel provided a continuous flow past the test circular cylinder by recirculating about 8,000 gallons of water driven by a four-bladed impeller connected to the drive motor. The speed of the drive motor was controlled by a variable frequency threephase drive, and had maximum rotational speed of 1750rpm. The channel water speed range resulting in VIV was $0.4 \mathrm{~m} / \mathrm{s}-1.5 \mathrm{~m} / \mathrm{s}$. The corresponding reduced velocity and Reynolds number were about $4 \leq U^{*} \leq 15$ and $3 \times 10^{4} \leq \mathrm{Re} \leq 1.2 \times 10^{5}$, respectively. The test section of the channel was $2.44 \mathrm{~m}$ long, $1 \mathrm{~m}$ wide, and $0.8 \mathrm{~m}$ deep. The honeycomb made flow straight and the screens result in uniform and low turbulence flow in the test section. Velocity and pressure were measured by pitot-static tube. Over the entire cross-sectional area in the test section, the streamwise flow was uniform to within $1.4 \%$ root mean square (RMS). Detailed information about the LTFSW Channel has been published by Walker et al. (1996).

A smooth circular cylinder of diameter $D=3.5 "(8.89 \mathrm{~cm})$ and length $L=36 "(91.4 \mathrm{~cm})$ was used for the experiments. An assessment of blockage effects was provided by Chang et al. (2011). As shown in Fig. 2(a), the test-cylinder was mounted on two linear endsprings and the cylinder motion was allowed in the cross-flow direction only. This one degree-of-freedom model provides useful information relevant to the more complex 
problem of two degree-of-freedom FIM of flexible cylinders, such as cables and marine risers, where the cylinder can oscillate in cross-flow and in-line.

The spring constant $k$ and damping coefficient $c$ were calculated using the logarithmic decrement method by a series of ten free-decay tests in air. It was assumed that the system damping ratio in air $\left(\zeta=\frac{c}{2 \sqrt{K m_{\text {osc }}}}\right)$ can be adequately represented by a linear viscous model. The source of the damping in the experimental device was only due to the friction between the linear bearings and the shafts. From the measured oscillating mass and the calculated spring constant, the natural frequency of the system in water was derived using a potential added mass coefficient, which is unity. To the oscillating mass of the cylinder, the oscillating spring-mass (effective mass) was added. It is calculated by dividing the spring into infinitesimal springs, finding infinitesimal force at each spring and the corresponding extension, and integrating the force. The resulting equivalent mass of the spring oscillating with the cylinder degree of freedom is $1 / 3$ of the spring mass. The results are shown in Table 1 including an error estimate based on the ratio of the standard deviation to the mean value.

The cylinder oscillatory displacement was measured by Celesco Inc. Model PT101 position transducer. The accuracy of the position transducer is $0.15 \%$ for full stroke. All the data were recorded at a $100 \mathrm{~Hz}$ sampling rate with duration of $60 \mathrm{sec}$. For amplitude plots, the average of the 60 largest positive and negative amplitude values were averaged. To calculate the oscillation frequency of the cylinder, time histories of the measured displacement were processed by Discrete Fourier Transform (DFT) to produce displacement spectra. The peak frequency was selected as the cylinder oscillation 
frequency. The frequency resolution was $1 / 60 \mathrm{~Hz}$, which was small enough to distinguish between important frequencies in the spectrum.

Passive Turbulence Control (PTC) was selectively distributed surface roughness in the form of two straight roughness strips. The roughness strips used in this paper were commercially available and designated as P180. Two straight roughness strips were placed symmetrically on the cylinder surface with respect to the flow, running along the entire cylinder span. In the present study, the PTC width was designed to cover $16^{\circ}$ of the circumference length of the circular cylinder. The effect of different width was studied and shown in Park et al. (2013b). PTC was applied at various circumferential locations of the circular cylinder. The location of PTC was defined by the leading edge angle $(\alpha)$ of the strip and stated in the legends of the amplitude and frequency figures. Fig. 2(b) shows PTC location and coverage on the circular cylinder surface. For example, P180: $60^{\circ}-76^{\circ}$ indicates $\alpha=60^{\circ}$ and coverage area of $16^{\circ}$. One strip of roughness P180 is placed with its upstream edge at $60^{\circ}$ and downstream edge at $76^{\circ}$ measured from the upstream ideal flow stagnation point at $\alpha=0^{\circ}$. The second roughness strip is placed symmetrically with its upstream edge at $-60^{\circ}$ and its downstream edge at $-76^{\circ}$.

The height of the grit and total thickness of the roughness strip $(H)$ are two important parameters. The total thickness of the roughness strip $(H)$ is the average height of the grit ( $k$ ) plus the height of backup paper ( $p$ ) plus the thickness of the double-sided tape $(192 \mu \mathrm{m})$ used to attach the PTC to the cylinder surface. The ratio of average roughness (k) height to the cylinder diameter $(D)$ is $92 \times 10^{-5}$. The ratio of total roughness strip $(H)$ thickness to the cylinder diameter $(D)$ is $645 \times 10^{-5}$. The total thickness of roughness chosen in the tests was on the order of the boundary layer thickness (Chang. et al. 2011). 
Broad Filed-Of-View (FOV) visualization was used to capture wake length of several diameters in order to understand the flow patterns and vortex structures around the oscillating cylinder. A 5W continuous laser beam emitted from an argon-ion laser, Coherent model I70C, was redirected and spread through a system of lenses and reflectors to obtain a uniformly distributed laser light sheet. The formed laser sheet illuminated the mid-plane perpendicular to the cylinder axis. Appropriate amount of aluminum oxide particles of 100 microns in size was uniformly dispensed through a tube with a series of small perforations resulting in clear flow visualization around the cylinder. A high-resolution black and white CCD camera, Imperx Inc, IPX-2M30H-L, which records the flow with maximum pixel resolution of $1920 \times 1080$ at $33 \mathrm{~Hz}$, was used for capturing the flow visualization images. At each flow velocity, the shutter speed of the camera was adjusted to optimize the image quality in the test area thus changing effectively the frame rate of the camera between $20 \mathrm{~Hz}$ and $30 \mathrm{~Hz}$. This rate was high enough to avoid aliasing compared to the highest frequency of cylinder oscillation observed, which was around 1.3-1.4Hz. Overall, the number of frames per cycle was 3080 making it easy to identify the location of the cylinder in a given cycle with accuracy.

\section{Smooth cylinder response}

In all amplitude and frequency response figures for PTC-cylinders presented in this paper, the corresponding smooth cylinder results are plotted for comparison. Results are plotted vs. Reynolds number (Re), and reduced velocity $\left(U^{*}=\frac{U}{f_{n, w} D}\right)$. The definition of the natural frequency in water $\left(f_{n, w}\right)$ is disputed as being non-constant and depending on 
added mass, which varies with $U^{*}$ (Sarpkaya 1979; Vikestad et al. 2000). In this paper, the reduced velocity is defined by the natural frequency in still water

$$
f_{n, w}=\frac{1}{2} \sqrt{\frac{K}{m_{o s c}+m_{A}}} \text {, where } K=\text { spring constant, } m_{o s c}=\text { oscillating mass, } m_{A}=\text { ideal }
$$

added mass. This affects the presentation of results but not their accuracy It is the authors' opinion that this controversy is unnecessary as both are simple modeling methods that cannot account for the range of synchronization or the self-limiting nature of the amplitude of oscillation. Both just model the response of the cylinder in FIM for a specific flow velocity and a given experiment. On the other hand, both approaches are useful in post-processing data and understanding this complex phenomenon better. For better understanding of the complexity of the added mass expression in forces and moments on a small body moving in a 3-D unsteady flow, the reader is referred to the detailed analysis by Foulhoux and Bernitsas (1993). The dependence of the inertia terms on absolute and/or relative velocity as well as the form of convective terms is explained thoroughly putting into perspective the simplicity of the inertia term used in Morison's equation

3.1. Amplitude and frequency response of smooth cylinder

Amplitude response for smooth cylinder: As seen in Fig. 3, the smooth cylinder is set into vibration at a reduced velocity of about 4.3. After a gradual build-up in the initial branch, $4.5<U^{*}<5.6$, amplitude increases abruptly between $U^{* \cong 5.6 \text { and }} U^{* \cong 5.8 \text { reaching }}$ the upper branch at $U^{* \cong 5}$.8. Upper branch response extends up to a reduced velocity of 


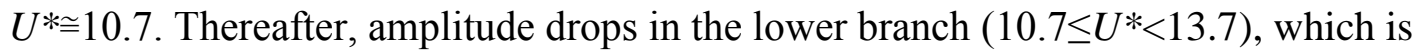
followed by desynchronization.

It should be pointed out that, in the present study for $3 \times 10^{4} \leq \mathrm{Re} \leq 1.2 \times 10^{5}$ the three branches differ in range and amplitude from those reported by Khalak and Williamson (1999), and Govardhan and Williamson $(2000,2006)$ for $3,500<\operatorname{Re}<10,000$. The upper branch in the present study is the broadest branch of the VIV synchronization range and the branch where the highest amplitude is observed. The peak amplitude of the upper branch in the present study is much higher than that at the lower Reynolds number. The tests performed in the present study are in the TrSL3 regime and the peak amplitude in the upper branch depends on Reynolds number. In the TrSL3 regime, formation length decreases and two-dimensional Reynolds stress level increases (Williamson 1996; Zdravkovich 1997; Govardhan and Williamson 2006). Hence, more coherent vortices are formed closer to the cylinder causing larger lift forces on the cylinder.

Govardhan and Williamson (2006) and Triantafyllou et al. (2004) concluded that there is dependence of the peak amplitude in VIV on Reynolds number by studying results for $\operatorname{Re}<33,000$. Bernitsas et al. (2006) and Raghavan and Bernitsas (2011) have shown the strong dependence of VIV amplitude on Reynolds number with tests in the TrSL3 regime reaching amplitudes to diameter ratio of 1.9 for Re about 125,000.

Furthermore, the lower branch in the present study is very narrow and has been overtaken by the upper branch in comparison to low Re VIV. The response in the lower branch is classified as a 'mixed response' falling between the conventional (low Re) lower branch with steady periodic oscillations and desynchronization characterized by random on-off VIV time-intervals. The lower branch in this study exhibits the characteristics of a 
conventional steady lower branch and desynchronization. This is the effect of much higher Reynolds numbers used in the present investigation.

Frequency response for smooth cylinder: In Fig. 3, along with the abrupt increase in amplitude, the frequency ratio $f^{*}=f_{o s d} / f_{n, w}$, where $f_{o s c}$ body oscillation frequency, also registers a step-increase at $U^{* \cong 5.6 . ~ I n ~ t h e ~ u p p e r ~ b r a n c h, ~ f r e q u e n c y ~ i n c r e a s e s ~ a l m o s t ~}$ linearly $\left(0.9 \leq f^{*} \leq 1.1\right)$. Thereafter $f^{*}$ undergoes a steep increase to a higher value of $f^{*} \cong$ 1.30 till $U^{* \cong 12.0}$ and remains nearly constant after $U^{* \simeq 12.0}$

Time histories and spectra for smooth cylinder: Fig. 4 shows the time histories of displacement in each branch and the corresponding power spectra. At the end of the initial branch, $U^{*}=5.6$, the displacement time history in Fig. 4(a) shows periodic oscillations with certain degree of modulation. The corresponding frequency spectrum shows only one predominant spike at the oscillation frequency $f_{o s c}=0.84 \mathrm{~Hz}\left(f^{*}=0.753\right)$. At $m *=2.4$, Khalak and Williamson (1996) also observed similar amplitude quasi-periodic wave-forms in the initial branch. However, results at still higher $m^{*}=8.63$ show much more stable and steady amplitudes in the initial branch (Fig. 4 of Govardhan and Williamson 2000). When $U^{*}$ is further increased to $U^{*}=8.56$ in the upper branch (Fig. 4(b)), variation in amplitude is further decreased and cylinder oscillations are steady and periodic. The frequency spectrum shows additional spikes at $2 f_{o s c}$ and $3 f_{\text {osc }}$. In the upper branch at $m^{*}=2.4$, Khalak and Williamson (1996) observed notable amplitude modulations in their Figs. 11(a)-(ii). In the lower branch at $U^{*}=13.4$ in Fig. 4(c), the vibrations are not steady exhibiting greater modulations in contrast to the nearly steady oscillations reported by Khalak and Williamson (1996), and as shown in Fig. 3(a), amplitude decreases steadily in contrast to the nearly flat and broader profile observed by 
Khalak and Williamson (1999). At $U^{*}=14.89$ (desynchronization), only feeble oscillations are observed and the corresponding spectrum is nearly uniformly distributed with no predominant wake frequencies as shown in Fig. 4(d).

3.2. Near-wake vortex structures for smooth cylinder at $3 \times 10^{4} \leq \mathrm{Re} \leq 1.2 \times 10^{5}$

Near-wake vortex structures around the smooth cylinder are presented in this section. These are made out of the frame-by-frame analysis of the flow visualization videos made as a part of the present study. The flow structures are captured for cylinder motion over a complete cycle of oscillation starting from the Bottom Dead Center (BDC), which refers to the lowest position of the cylinder in an oscillatory cycle, to the Top Dead Center (TDC) and back to BDC. The phase of cylinder motion is expressed in terms of $\mathrm{t} / \mathrm{T}$, where $\mathrm{t}$ is real time and $\mathrm{T}$ is the period of oscillation. Only the salient features of the flow structures are illustrated. It should be noted that the sketches of flow patterns presented are drawn not to scale as they are primarily meant to bring out the qualitative aspects of the flow fields and not any quantitative measures. In all cases, the wake structures within a downstream distance of about $4.0 \mathrm{D}$ are captured. Of the sixty cycles recorded and photographed per test, a typical steady-state periodic cycle was analyzed to identify vortex structures. Several actual pictures are merged in the sketches in Figs. 5-8 for comparison. Actual flow visualization pictures follow the corresponding sketches. It is much easier for the reader to follow the vortex structure development by studying the sketch sequences than the actual pictures. The pictures help confirm the accuracy of the sketches.

\subsubsection{Initial branch}


Initial branch commences with the $2 \mathrm{~S}$ mode of shedding and it starts at $U^{*}=4.84$.

It is not presented here since it is a well-known mode of vortex shedding. As the reduced velocity is increased, at $U^{*}=5.6$, i.e. at the upper end of the initial branch, it is found that 3 vortices are shed per cycle of oscillation, and the non-dimensional amplitude $A *$ is 0.404. The vortex pattern observed in a typical oscillatory cycle is given in the sketch sequence in Fig. 5. The vortices are numbered (e.g., V1, V2), in the order of their initial formation while still attached to the cylinder. This order may be different from the order of shedding. Thick and short flow lines with arrows marked in the flow fields, as in Figs. 5(d) and 5(e), indicate flow of irrotational fluid entrained from the outer flow between two vortices of opposite vorticity. This irrotational fluid is needed in addition to the opposite vorticity flows from the two shear layers; all three meet at the confluence point for a vortex to shed per Gerrard's mechanism (1966). These thick short lines with arrows marked on the sketches can be observed in the visualization video (32 frames per second) but of course cannot be observed in individual pictures shown in Figs. 5-8.

As Fig. 5 shows, starting from BDC in Fig. 5(a), the bottom shear layer produces two vortices V2 and V3 (V3 appears at BDC, whereas V2 was formed earlier) coalescing to form a larger vortex V2,3 at $\mathrm{t} / \mathrm{T}=0.167$ in Fig. 5(c). Vortex V4 is initially formed at $\mathrm{t} / \mathrm{T}=0.084$ in Fig. 5(b) growing as the cylinder moves up towards TDC. V4 undergoes limited growth due to the presence of the stronger vortices V2,3 and V1 (formed in the previous cycle) in Fig. 5(b). V4, which rolls up weakly and dissipates quickly, is shed at t/T=0.375 in Fig. 5(f) due to the 'passive pushing' by its co-generated (same shear layer) vortex V5. This passive shedding mechanism was first observed for a square section cylinder (Kumar et al. 2009). After significant growth, V2,3 is shed at t/T=0.292 in Fig. 
5(e). Shedding of V2,3 occurs due to the action of fluid carrying oppositely signed vorticity (following the mechanism described by Gerrard 1966). At t/T=0.458 in Fig. 5(g), V6 forms at the bottom shear layer. At TDC, a new vortex V7 forms from the top shear layer (Fig. 5(h)) and coalesces with V5 to form V5,7 at t/T=0.667 in Fig. 5(k). V6 formed at $\mathrm{t} / \mathrm{T}=0.458$ steadily grows and rolls over the cylinder towards its base reaching bigger than usual size and shedding in the next cycle after V5,7. Vortex V5,7 sheds at $\mathrm{t} / \mathrm{T}=0.875$ in Fig. 5(n). Hence, 3 vortices (V2,3, V4, and V5,7) shed per oscillatory cycle with no pairing between the shed vortices; for $\mathrm{Re} \cong 43,300$ and $A^{*} \cong 0.4$. This shedding pattern is different from the $2 \mathrm{~S}$ mode observed by Williamson and Roshko (1988) for $\mathrm{Re}$ $\cong 1,000$ and $A$ * $\cong 0.4$. and extended to $\operatorname{Re} \cong 3,500-10,000$ (Khalak and Williamson, 1999).

The wake inclination angle in Figs. 5(i)-(k) clearly does not follow the direction of the relative flow velocity. Starting from TDC in Fig. 5(h), it takes about $0.25 \mathrm{~T}$ for the wake to align itself to the direction of the relative flow velocity. This wake swing is due to the existence of wake inertia.

\subsubsection{Upper branch}

As the reduced velocity is increased, the upper branch is reached and different vortex patterns are observed compared to the initial branch. Fig. 6 shows flow structure $2 \mathrm{P}$ around the cylinder at $U^{*}=8.18$ for $\operatorname{Re}=63,500$ and $A^{*}=1.39$.

Starting from BDC in Fig. 6(a), during the upward travel of the cylinder, vortices V1 (shed at $\mathrm{t} / \mathrm{T}=0.278$ in Fig. 6(d)) and V2 (shed at $\mathrm{t} / \mathrm{T}=0.111$ in Fig. 6(b)) form a first pair at $\mathrm{t} / \mathrm{T}=0.278$ in Fig. 6(d). A second pair of vortices is formed by V3 (shed at $\mathrm{t} / \mathrm{T}=0.723$ in Fig. $6(\mathrm{~h})$ ) and $\mathrm{V} 4$ (shed at $\mathrm{t} / \mathrm{T}=0.50$ in Fig. $6(\mathrm{f})$ ) at $\mathrm{t} / \mathrm{T}=0.723$. In this case, 
vortex V4 is shed by the action of oppositely signed vorticity, i.e. following Gerrard's mechanism, whereas V1 and V2 are shed due to the operation of both 'passive pushing' mechanism and Gerrard's mechanism. For V3, passive pushing by V6 appears to be the sole mechanism inducing shedding. Thus, the mode of vortex shedding observed is $2 \mathrm{P}$ (V1 to V4).

At a lower reduced velocity of $U^{*}=6.32$ in the upper branch, the same $2 \mathrm{P}$ mode of shedding is observed for the majority of the oscillatory cycles but without vortex pairing in few cycles of oscillation. That is, in few oscillatory cycles, vortices are not pairing up possibly due to the difference in their convection velocities. A close examination of flow structures reveals that vortices with near-equal convection velocities pair-up provided their shedding timings are appropriate. In addition to these facts, vortex-vortex interactions appear to be influenced by wake deflections.

In addition to the $2 \mathrm{P}$ mode, the $3 \mathrm{P}$ mode of shedding is observed in a few oscillatory cycles. That is, an additional pair of vortices is shed (either by the top or the bottom shear layer) in some cycles of oscillation giving rise to the 3P mode of shedding. These two additional vortices are shown in Fig. 6(k), where an additional pair of vortices is formed in the bottom shear layer contributing to the 3P mode in one cycle. On closer inspection, it is also noticed that, in those cycles with 3P shedding mode, the amount of vortices are in generally larger (stronger) when compared to those in $2 \mathrm{P}$ mode. Thus, it is conjectured that the amount of vorticity generated and shed is higher, which is rightly reflected in the higher amplitude of oscillation observed in the upper branch of the present study. 
The vortex pairs after formation are observed to traverse in the direction of the wake axis (which is skewed many a times due to wake deflections). It is pointed out that, in a vortex pair, the sizes of participating vortices are not always the same. Many a times, they are identical but sometimes, notable size difference is observed between them. In the upper branch, at higher reduced velocities, higher wake deflections (wake swings) are observed. Such wake swings (but with lower angle of swing) have been reported by Ongoren and Rockwell (1988) also even at lower Reynolds numbers.

Body acceleration could be a major factor in the formation of new vortices (Honji and Taneda 1969; Williamson and Roshko 1988). This is confirmed in the present study by Fig. 6(b) where new vortex V3 forms near the maximum acceleration point. On the other hand, results show that new vortices need not be generated at the maximum acceleration position (BDC/TDC) of the cylinder travel. Instead, vorticity roll up may occur at cylinder positions other than BDC/TDC e.g., Fig. 6(d) for V4 and Fig. 6(i) for V5 and V6.

It should be noted that, the 2Pand 3P modes in the upper branch at $U^{*} \geq 8.2$ observed in this work for $3 \times 10^{4} \leq \mathrm{Re} \leq 1.2 \times 10^{5}$ fall in the 'no-synchronized pattern zone' of the Williamson-Roshko map for 300<Re $<1,000$ (Fig. 3(a) of Williamson and Roshko 1988). Recently, Morse and Williamson (2009) employed controlled vibration to predict response and wake modes of the free vibration of cylinders. With 5,680 runs they compiled high-resolution contour plots of the lift force on the map of the regimes of the vortex-structures and the controlled vibration results are in very close agreement with free vibration results. At $\operatorname{Re}=4,000$ and 12,000 with $m^{*}=10$, a remarkable similarity between the vortex formation mode boundaries from controlled vibration by Morse and 
Williamson (2009) and flow visualization by Williamson and Roshko (1988) map is

found. In the updated map, the $2 \mathrm{P}$ and $3 \mathrm{P}$ modes of the present study still fall in the "nosynchronized pattern zone'. They point out, however, the possibility of vortex-induced motion in the desynchronized wake region if there is some positive fluid excitation at the oscillation frequency.

Direct comparison though is not possible between the results of the present work and the Morse and Williamson updated map due to the large difference in Reynolds number and mass ratio $m^{*}$. Re is an order of magnitude higher in the present work and experiments are conducted in the TrSL3 flow regime where the shear layer is fully saturated, vortex strength is stronger, and formation-length is shorter resulting in much higher lift coefficient (Zdravkovich 1997).

\subsubsection{Lower branch}

In the lower branch, a periodic vortex shedding pattern is not observed, and notable cycle-to-cycle variations are observed in the wake vortex structures (Fig. 7). This variation is due to the fact that the vortex shedding process starts to unlock from the cylinder motion. For the majority of cycles, about 3 to 7 individual vortices are shed per cycle of oscillation; vortex shedding frequency varies from cycle to cycle. Frequently, two vortices symmetrically attached to the cylinder are developed as in Figs. 7(b), 7(c), 7(f), 7(h). These vortices are very weakly rolled up and are quickly dissipated in the wake. Fig. 7 shows flow structures behind the cylinder where seven individual vortices are shed per cycle at $U^{*}=12.28$. 
Vortex V1, formed in the previous cycle, sheds after the cylinder moves from BDC in Fig. 7(a). As the cylinder progresses in its upward motion from BDC (Fig. 7(b)), vortex V2 formed in the top shear layer grows and is shed by a combination of Gerrard's (1966) mechanism and the passive pushing mechanism. Vortex V3 formed in the bottom shear layer grows and is shed only by passive pushing mechanism. Before shedding, two vortices V2 and V3 attach symmetrically to the cylinder. The symmetrically attached vortices imply a nearly symmetric pressure distribution on the cylinder surface. This near equal pressure distribution causes low lift force (Gerrard 1966). Similarly to V2, and V3, two vortices V4 and V5 attach to the cylinder symmetrically in Fig. 7(c)). Vortex V5 sheds by the action of fluid carrying oppositely signed vorticity at t/T=0.512 in Fig. 7(d) per Gerrard's mechanism and vortex V4 sheds by a combination of Gerrard's mechanism and passive pushing at $\mathrm{t} / \mathrm{T}=0.751$ in Fig. $7(\mathrm{e})$. Two vortices V6 and V7 grow at the same time at $\mathrm{t} / \mathrm{T}=0.751$ in Fig. $7(\mathrm{e})$. Vortices V8 and V9 also grow at the same time at $\mathrm{t} / \mathrm{T}=0.927$ in Fig. $7(\mathrm{f})$. Thus, out the seven vortices shedding per cycle, six shed in pairs which grow and shed nearly symmetrically resulting in low lift force.

As mentioned earlier, there is cycle-to-cycle variation in the wake structures as can be observed by comparing the following pairs of pictures which correspond to the same time in different cycles: Fig. 7(g) in cycle 2 to Fig.7(b) in cycle 1 and Fig.7(h) in cycle 3 to Fig. 7(d) in cycle 1 . These are three different cycles occurring during the same test at constant $U^{*}$. This observation indicates that the body oscillation is not synchronized with the vortex shedding process, which is clearly reflected in the corresponding displacement time histories of the body motion. 


\subsubsection{Desynchronization}

In desynchronization, coupling between the oscillating body and its wake appears to be lost almost completely. No definite periodicity is observed in the wake of the cylinder indicating that the shedding process is completely unlocked from the cylinder motion. In desynchronization, as shown in Fig. 8 at $U^{*}=14.5$, vortex shedding is completely suppressed in the range of Reynolds number studied in this paper. Instead of a von Kármán Street, two wake structures are observed. In Fig. 8(a), two attached vortices symmetric about the wake axis are developed. This results in near-equal pressure distribution on the top and bottom surfaces of the cylinder, inducing only negligible lift force and consequently, small amplitude motion. Generally in this case, the vortices are weakly rolled up and this rightly reflects the smaller excitation amplitudes in desynchronization. In Fig. 8(b), the cylinder wake appears to be extremely turbulent with no proper vortex roll up to excite the cylinder, which is consistent with the amplitude time history shown in Fig. 4d.

\section{Weak suppression zones (WS1 and WS2)}

A zone is defined as the range of the position of the angle $(\alpha)$ of the leading edge of the roughness strip, where similar FIM of the PTC-cylinder is observed. Experiments are repeated in small increments of angle $\alpha$ to establish the width of each zone. Weak suppression is defined as the case where the peak amplitude of oscillation is no less than $70 \%$ of the peak amplitude of the smooth cylinder. For P180 roughness strips, two zones of weak suppression (namely WS1 and WS2) have been identified. They are shown in 
Fig. 1(a) and designated as $0^{\circ} \leq \alpha<2^{\circ}$ (WS1) and $106^{\circ} \leq \alpha \leq 180^{\circ}$ (WS2), where angle $\alpha$ range indicates zone width.

4.1. Amplitude and frequency response in the weak suppression zones

Amplitude response in the WS1 and WS2 zones: As shown in Fig. 3(a), for the WS1 and WS2 zones, the amplitude response of the PTC-cylinder are not significantly different from those of the smooth cylinder except in the reduced velocity range $9.5 \leq U^{*} \leq 11.5$. Particularly, the initial and upper branches of WS1 and WS2 are identical to the initial and upper branches of the smooth cylinder, respectively. Onset of the initial branch is at $U^{* \approx 4} .5$ and of the upper branch at 5.8 for both smooth and PTC-cylinders. Especially the farther to the back of the cylinder the roughness strip is placed in WS2, the more ineffective PTC becomes resulting in increased amplitude and range of synchronization.

For all WS1 and WS2 zones, PTC configurations (varying the circumferential location) show less amplitude and shorter synchronization regions than those of the smooth cylinder. In the amplitude response, the difference between WS1 and WS2 is minor. For WS1, the PTC is located at the "ideal flow" stationary stagnation point. For WS2, the PTC is located far from a stationary or a separation point. Hence, the suppression mechanism is different and this mechanism is discussed in Section 4.2.

Frequency response in the WS1 and WS2 zones: As shown in Fig. 3(b), the frequency ratio $f^{*}=f_{o s c} d f_{n, w}$ for all weak-suppression cases follow that of the smooth cylinder and gradually increases up to around 1.3. The only difference between WS1, WS2, and 
smooth cylinder response is that WS1 has higher $f^{*}$ and WS2 has lower $f^{*}$ than that of the smooth cylinder for $6<U^{*}<10.7$ (upper branch of WS). In this range for WS2, higher strip angles result in higher frequency ratio. When the roughness strips are placed close to the base region of the circular cylinder, frequency response is very close to that of the smooth cylinder.

Time histories and spectra in the WS zone: The displacement time histories in WS2 are similar to those in WS1. The displacement time histories at different reduced velocities of WS1 are shown in Figs. 9(a)-(d). In the initial branch at $U^{*}=5.6$, oscillation is periodic with small modulation similar to that of the smooth cylinder in Fig. 4(a) The corresponding spectrum shows a significant spike at $f_{\text {osc }} \cong 0.86$. Then, at $U^{*}=8.56$ in the upper branch, significant oscillations are recorded as shown in Fig. 9(b). Spectrum shows a major spike, which corresponds to the oscillation frequency, and two minor spikes at the second and third harmonics. A weak lower branch follows the upper branch in the range $10.7 \leq U^{*} \leq 13.7$. Typical lower branch characteristics of WS1 are shown in Figs. 9(c). At $U^{*}=11.91$, amplitude modulation is observed indicating that motion is not completely steady and spectrum shows that displacement exhibits less periodicity at the oscillation frequency with absence of any higher harmonics. In desynchronization, weak and unsteady oscillations are recorded $\left(U^{*}=14.89\right.$, Fig. $\left.9(\mathrm{~d})\right)$.

4.2. Wake vortex structure in the weak suppression zones (WS1, WS2)

The flow field around the PTC-cylinder in the weak suppression zones (both WS1 and WS2) is studied. In the present visualization experiments, the strength of vortices is compared qualitatively. For example, a fully formed vortex, which is just about to shed 
from a smooth cylinder, was taken as the reference vortex with which the qualitative comparisons with other vortices are made. Comparisons are made between vortices for the same flow velocity, which is equal to the outer velocity of the vortex when ready to shed.

Using the simple Rankin vortex model, its circulation (strength) is $\Gamma=2 \pi R U$. For the same flow velocity $U$, circulation is proportional to the vortex radius $R$. Thus, in this study, vortices at the same velocity and shedding moment are compared visually for strength to explain change in lift and, thus, response amplitude at different scenarios such as a PTCcylinder versus a smooth cylinder.

Luo (1992) concluded that, in a visualized flow, the amount of circulation associated with a vortex can be approximately estimated from the size of the vortex for a given flow velocity. He directly correlated the vortex size to the circulation values estimated from flow visualization at the same flow speed. A larger vortex carries more circulation in it and hence is 'stronger'. Furthermore, Kumar \& Gowda (2006) and Gowda \& Kumar (2006) justified the enhancement and suppression of square cylinder vibrations based on the strength of shed vortices qualitatively assessed from their size and the quality of vortex roll up.

The wake structures in the initial, upper and lower branches are remarkably similar to their counterparts for a smooth cylinder at the same velocity but with weaker-smaller vortices (less circulation) when the strength of vortices is compared qualitatively.

In the WS1 case, the flow is separated at the upstream edge of the strip and reattaches as the boundary layer develops again on the cylinder surface since the strip location is close to the front stagnation point. This is shown in Fig. 10 using dye (Chang 2010). The boundary layer is more diffused (thicker) due to the presence of the roughness elements followed by reattachment of the boundary layer. This was also observed by Nebres and Batill (1993) using a wire as surface protrusion on a stationary cylinder with ratio of surface protrusion to cylinder diameter close to that of the present study. 
In WS1, the surface roughness affects the vortex formation length $l_{f}$, defined as the non-dimensional distance between the cylinder center and the center of the vortex at the moment of shedding (Krishnamoorthy et al. 2001) as shown in Fig. 11. The diffusion of the boundary layer due to the roughness elements leads to a small increase in the vortex formation length as shown in Fig. 11(b). This has been specifically verified for the WS1 case with configuration $\mathrm{P} 180: 0^{\circ}-8^{\circ}$ as shown in Fig. 3. For this configuration, at $U^{*}=10.7$, where the amplitude difference between the WS1 and the smooth cylinder is maximum, $l_{f}$ is about $7.5 \%$ longer for the PTC cylinder than that of the smooth cylinder at bottom dead center (BDC). This agrees with the observation by Lee and Kim (1997) that surface protrusion increases the vortex formation length of a circular cylinder.

For WS2, since the attached roughness is inside the base region of the cylinder, the boundary layer is not significantly disturbed. Instead, the roll up of shear layers is adversely affected leading to the formation of weaker and disorganized vortices, as shown in Fig. 11(c).

\section{Strong suppression zone (SS)}

Cases where the peak amplitude is suppressed by $30 \%$ or more - compared to the peak amplitude of the smooth cylinder response - are classified as strong suppression cases. For P180, the strong suppression zone is located in the range $60^{\circ} \leq \alpha<106^{\circ}$.

5.1. Amplitude and frequency response in the strong suppression zone

Amplitude response in the SS zone: Fig. 12(a) shows the amplitude in strong suppression 
cases. Most prominent amplitude reduction is observed when P180 is placed at $70^{\circ}$. For P180: $70^{\circ}-86^{\circ}$, the amplitude drops to negligible levels at $U^{* \cong 7.0 ~ a n d ~ i s ~ n e a r l y ~ f u l l y ~}$ suppressed for $U^{*} \geq 13$. A high amplitude $\left(5.8<U^{*}<6.8\right)$ region and a lower amplitude $\left(6.8<U^{*}<12.5\right)$ region corresponding to the upper and lower branch, respectively, are present. Then, desynchronization $\left(12.5<U^{*}\right)$ follows. For this case, the synchronization range and maximum amplitude are reduced more than 50\% compared to those of the smooth cylinder. For all cases in the strip angle range $\left(80^{\circ} \leq \alpha \leq 90^{\circ}\right)$, lower branch persists up to $U^{* \cong 15.0}$ with smaller but notable amplitudes even at $U^{*}=14.0$.

Explanation for the strong amplitude suppression follows. First of all, in this work, suppression is not due to transition from laminar to turbulent flow. Roughness can induce transition from laminar to turbulent at lower Reynolds number (Achenbach 1971; Achenbach and Heinecke 1981). It is a well-known experimental fact that in transition, VIV is fully suppressed (Tritton 1977). The following two facts prove Re is still low and transition has not been reached: (a) At the same Reynolds number we can enhance the FIM response (Chang et al. 2011; Park et al. 2013a) by moving the PTC location based on the PTC-to-FIM Map in Fig. 1. (b) If the experiments had reached critical flow there would be total suppression of VIV, which is not the case in Fig. 12.

The reason for the strong suppression is the following. In VIV, the lift force is proportional to the strength (circulation) of the vortices. The high response of the smooth cylinder in the present work lies in the fact that tests are conducted in the TrSL3 flow regime where the shear layers are fully saturated contributing maximum circulation to the large shed vortices. Hence, the placement of the PTC for suppression, aims to destroy the shear layer vorticity before it rolls up into the vortices. This is a matter of finding 
experimentally the right thickness of roughness with respect to the boundary layer, as well as the right roughness, right starting, and right ending points - a total of four parameters. When the strip upstream-edge is located around $60^{\circ}-70^{\circ}$, VIV is suppressed everywhere but in a very narrow range of reduced velocity. This range of the roughness strip covers the smooth cylinder separation point in laminar flow (around $81^{\circ}$ ) and the surface around it within its oscillation range of about $\pm 5^{\circ}-10^{\circ}$ due to vortex shedding. The weaker vortices can still support VIV in the narrow range $6<U^{*}<7$ (P180: $70^{\circ}-86^{\circ}$ in Fig. 12(a)) because it is too close to the natural frequency of the oscillator and thus hard to fully suppress.

Frequency response in the SS zone: A typical frequency response in strong suppression is shown in Fig. 12(b). The narrow-high amplitude region in Fig. 12(a) is close to the natural frequency of the system in still water. As shown in Fig. 12(b), the frequency ratio is close to unity for $5.8<U^{*}<6.8$. The strongest suppression takes place for P180: $70^{\circ}-86^{\circ}$ and for $U^{*>7.8 . ~ I n ~ t h i s ~ c a s e ~ t h e r e ~ i s ~ n o ~ p r e d o m i n a n t ~ f r e q u e n c y ~}$ observed. For the larger strip location angles $\left(80^{\circ} \leq \alpha \leq 100^{\circ}\right)$, at the end of the lower branch, the frequency response is high while at lower angles and for the smooth cylinder there are no oscillations. The larger the strip placement angle within the range $80^{\circ} \leq \alpha \leq 100^{\circ}$, the higher is the frequency of oscillation for $U^{*}>8.5$. Particularly for $U^{*}>10.0$, the frequency ratio keeps increasing and for $U^{*}>13.0$ the cylinder exhibits much higher oscillation frequency compared to the smooth cylinder reaching up to $f^{* \cong 2.1}$

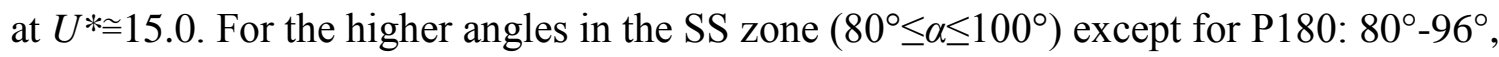
frequency change is gradual. The frequency jump found in the smooth cylinder from initial to upper branch is not observed here. 
Time histories and spectra in the SS zone: Fig. 13 shows displacement time histories of strong suppression at P180: $70^{\circ}-86^{\circ}$. Figs. 13(a) shows the response in the initial branch at $U^{* \cong 4}$.84. Corresponding spectra show a dominant frequency spike indicating the oscillation frequency. Similarly to smooth cylinder and WS response, oscillation has

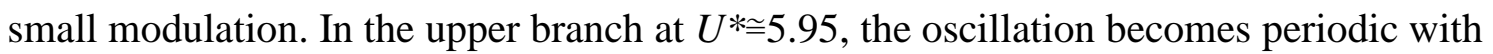
less modulation and amplitude than those of the smooth cylinder. In the lower branch at

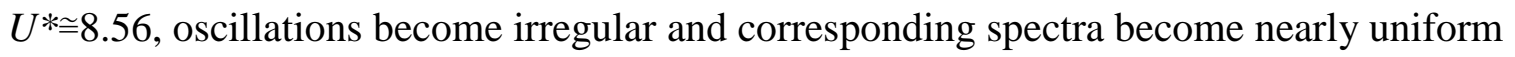
with no predominant spikes as shown in Fig. 13(c). In desynchronization at $U^{* \cong 13.0 ~ i n ~}$ Fig. 13(d), oscillation is insignificant. In each branch in the SS zone, the time history of displacement is similar to that of the smooth cylinder but with significantly lower amplitude except for P180: $90^{\circ}-106^{\circ}$ and P180: $100^{\circ}-116^{\circ}$ at $14 \leq U^{*}$. For P180: $90^{\circ}-106^{\circ}$ at $14 \leq U^{*}$, regular vortex shedding with high frequency is reappeared after the lower branch as shown in Fig. 13(e). Due to the LTFSW Channel limitation, amplitude response at higher reduced velocity could not be investigated.

5.2.Wake vortex structures in the strong suppression zone (SS)

Wake vortex structures in strong suppression using configuration P180: $70^{\circ}-86^{\circ}$ are

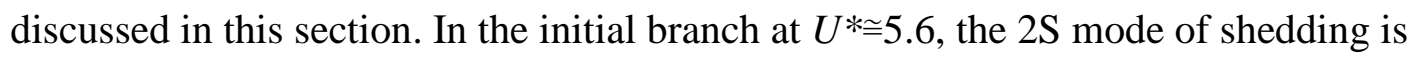
found but with $l_{f}$ increased by about $64 \%$ compared to the smooth cylinder as shown in Fig. 14. At $U^{* \cong 6} .32$ corresponding to the upper branch, the $2 \mathrm{P}$ mode of shedding appears in the near-wake with vortex size to diameter ratio being notably smaller than that of the smooth cylinder at the same $U^{*}$ value. At $6.7<U^{*}<12.5$, which falls in the stronger 
suppression region, no regular shedding is observed. Also, the wake is tapering in the downstream direction with its width substantially reduced. During most of the cycle, the wake is comprised of two weaker (less circulation) attached vortices similar to what is shown in Fig. 8(a). This is similar to the case of smooth cylinder desynchronization. As discussed in 3.2, this total circulation reduction results in proportional drop in the lift force. This lift force drop reflects amplitude reduction in the SS zone.

At $U^{*>} 12.5$ where the cylinder motion is negligible, sometimes two weak symmetric vortices are observed (Fig. 8(a)) but most of the generated vorticity from the shear layers diffuses in the wake in the form of irregular, small-scale eddies (Fig. 8(b)). This wake vortex structure shows the cylinder near-wake confined between two flow lines forming a foil-like hydrodynamic tail with small eddies escaping into a narrow wake. In general, a bluff body has a wide wake region and its form drag is dominant compared to skin friction. A streamlined body such as an airfoil has a narrow wake region and skin friction is dominant compared to form drag since the separation point of the streamlined body is much delayed compared to that of the bluff body. In the present study, the wake pattern of the PTC-cylinder, which is a bluff body, is similar to the streamlined body and the form drag is much decreased compared to the smooth cylinder.

Comparing wake length at the desynchronization of the smooth cylinder with the strong suppression PTC case at the same $U^{*}=14.51$, strong suppression induces longer wake length than that of the smooth cylinder by up to 55\%. According to Zdravkovich (1981), in cylinder-flows with good suppression devices, vortices are not dissipated but are formed farther downstream

A closer inspection revealed that shear layers fail to achieve proper roll up. According 
to Gerrard (1966), this possibility of symmetric vortex configuration is possible only at a Reynolds number of the order of $10^{3}$. In the range of Re considered in this paper, this phenomenon is observed both in smooth cylinder desynchronization and in strong suppression of the PTC-cylinder. In these cases most of the times, the wake is made up of two weak, attached vortices and irregular small scale eddies as shown in Fig. 8. Gerrard (1966) also pointed out that such symmetric vortex configurations would lead to a reduction of lift force, which is in agreement with the suppression results in the strong suppression zone.

For the configuration P180: $90^{\circ}-106^{\circ}$ (Fig. 12(a)), 2S and 2P mode of shedding is observed in the initial (at $U^{* \cong 5.7)}$ and upper branch (at $U^{* \cong 8.39), ~ r e s p e c t i v e l y, ~ b u t ~ w i t h ~}$ smaller vortex size to cylinder diameter ratio than in the smooth cylinder case. As the reduced velocity increased in the upper branch at $U^{* \cong 10.68, ~ a p a r t ~ f r o m ~ t h e ~} 2 \mathrm{P}$ mode, irregular symmetric vortex formation is also observed many times. At a still higher reduced velocitiy of $U * \cong 14.87$, symmetric vortex formation becomes the predominant wake vortex structures with occasional $2 \mathrm{~S}$ shedding mode. Substantial reduction in the wake width and downstream extent of the wake is observed. Symmetric vortex formation is thought to be due to intermittent, non-uniform re-attachment of the shear layers (Higuchi et al. 1989).

\section{Conclusions}

In this paper, the effect of local surface roughness (PTC) on a cylinder free to oscillate transversely to a flow has been studied experimentally. The corresponding smooth cylinder has also been tested for comparison. All experiments were conducted in 
the LTFSW Channel at the MRELab at the University of Michigan in the range $3 \times 10^{4} \leq \operatorname{Re} \leq 1.2 \times 10^{5}$. This range is in flow regime TrSL3, which is the high-lift, fully saturated shear-layer regime. The PTC location was varied along the circumference of the cylinder while all other experimental conditions were kept constant.

The major conclusions are the following:

1. Depending on PTC location, two weak suppression zones (WS1 and WS2) and one strong suppression zone (SS) were identified. Strong suppression (SS) was defined arbitrarily as at least $30 \%$ reduction in peak amplitude compared to the smooth cylinder response at the same $m^{*}$ and Reynolds number. SS occurs when the beginning of the PTC is located in the range $60^{\circ}<\alpha<106^{\circ}$.

2. For P180: $70^{\circ}-86^{\circ}$ in the SS zone, a single dominant frequency is observed only in a narrow region close to the natural frequency of the system in still water. The corresponding amplitude is about half that of the smooth cylinder. Elsewhere amplitude is nearly suppressed thus reducing the synchronization range from $5<U^{*}<12$ to $6<U^{*}<7$. 3. For P180: $70^{\circ}-86^{\circ}$ in the SS zone, for $U^{*}>7.5$, two new wake vortex structures were observed consistent with the amplitude reduction observation. The first wake vortex structure shows two weaker vortices remaining attached to the cylinder while additional vortices is diffused through a narrow wake. The second wake vortex structure consists of irregular, small-scale eddies diffused through a narrow wake. In both cases, the cylinder with its near wake takes the form of a symmetric airfoil.

4. In the WS1 and WS2 zones, the response features of the PTC-cylinder are similar to those of the smooth cylinder but exhibit smaller amplitudes. The onsets of the initial and upper branches in weak suppression are identical to those of the smooth cylinder. The 
only exception is that the frequency ratio $f^{*}$ is higher in the WS 1 zone and lower in the WS2 zone with respect to $f^{*}$ in the upper branch of the smooth cylinder.

5. Flow visualization in the WS zones showed that the vortex formation length is longer and vortices are weaker-smaller than those of the smooth cylinder even though the vortex structures are similar at identical reduced velocity values. Diffusion of boundary layer and improper shear layer roll-up cause the drop in response amplitude. However, the cause of longer vortex formation length would be different for WS1 and WS2. In the WS1 case, the flow separated at the upstream edge of the strip reattaches and the boundary layer is more diffused (thicker) due to interaction with the roughness elements. For WS2, the roll up of shear layers is adversely affected by PTC even though PTC is located downstream of the separation point.

6. For the smooth cylinder in the present study, the VIV response at high Reynolds number $\left(\operatorname{Re} \cong 10^{5}\right)$ is qualitatively different from VIV response at lower Reynolds number $(\operatorname{Re} \cong 4,000-12,000)$. The upper branch in the present study is broad with peak amplitudes exceeding $A^{*}=1.6$ and overtaking the lower branch. This is due to the high-lift in the TrSL3 flow regime. This high peak amplitude verified the fact that as Reynolds increases, the peak amplitude of the upper branch increases. The lower branch in the present study is narrow and has characteristics of desynchronization. That is, non-steady oscillations are observed unlike stable and steady oscillations reported in the literature for $\operatorname{Re} \cong 10^{4}$

7. For the smooth cylinder, vortex structures assumed conventional patterns $2 \mathrm{P}$ and $2 \mathrm{~S}$, and some non-conventional patterns as follows: At the onset of the initial branch, $2 \mathrm{~S}$ is observed while at the end of the initial branch three individual vortices are shed per 
oscillatory cycle. In the upper branch, the $2 \mathrm{P}$ pattern is observed. Occasionally, $3 \mathrm{P}$ is observed in the upper branch with two pairs on the same side thus maintaining the synchronization between the cylinder oscillation and the wake frequency. In the lower branch, 3-7 vortices are shed per cycle with notable cycle-to-cycle variation in the nearwake structures.

\section{Acknowledgements}

The following support is gratefully acknowledged: DOE contract DE-EE0003644 to Vortex Hydro Energy with subcontract to the University of Michigan.

\section{References}

Achenbach, E. 1971. Influence of surface roughness on the cross-flow around a circular cylinder. Journal of Fluid Mechanics 46, 321-335.

Achenbach, E. Heinecke, E. 1981. On vortex shedding from smooth and rough cylinders in the range of Reynolds numbers $6 \times 10^{3}$ to $5 \times 10^{6}$.Journal of Fluid Mechanics 109, 239251.

Assi, G.R.S., Bearman, P.W., Kitney N. 2009. Low drag solutions for suppression VIV for circular cylinders. Journal of Fluids and Structures 25, 666-675

Baarholm, G.S., Laresn, C.M., Lie, H. 2006. On fatigue damage accumulation from inline and cross-flow vortex-induced vibrations on risers. Journal of Fluids and Structures 22-1, 109-127.

Bearman, P. W. 1984. Vortex shedding from oscillating bluff bodies. Annual Review of Fluid Mechanics 16, 195-222.

Bernitsas, M. M., Ben-Simon, Y., Raghavan, K.,Garcia, E. M. H. 2006. The VIVACE converter: Model tests at Reynolds numbers around $10^{5}$. In $26^{\text {th }}$ Conference on Ocean, Offshore, and Artic Engineering, Hamburg, Germany, June 2006; also ASME Journal of Offshore Mechanics and Arctic Engineering 2009, 131-1, 1-13.

Bernitsas, M. M., Raghavan, K. 2008. Reduction/suppression of VIV of circular cylinders through roughness distribution at $8 \times 10^{3}<\operatorname{Re}<1.5 \times 10^{5}$. Proceedings of OMAE2008, Estoril, Portugal, June 15-20. 
Bernitsas, M. M., Raghavan, K. 2011. Enhancement of vortex induced forces \& motion through surface roughness control. United States Patent and Trademark Office, Patent\# 8,042,232 November 1, 2011.

Blevins, R. D. 1990. Flow-induced vibration, 1st ed. Van Nostrand Reinhold, New York.

Chang, C.C., Hydrokinetic energy harnessing by enhancement of flow induced motion using passive turbulence control. Ph.D. Dissertation, Dept. of Naval Architecture and Marine Engineering, University of Michigan 2010.

Chang, C. C., Kumar, R. A., Bernitsas M. M. 2011. VIV and galloping of single circular cylinder with surface roughness at $3.0 \times 10^{4} \leq \mathrm{Re} \leq 1.2 \times 10^{5}$. Ocean Engineering 38-16, 1713-1732.

Every, M. J., King, R.,Weaver, D. S. 1982. Vortex-excited vibrations of cylinders and cables and their suppression. Ocean Engineering9,135-157.

Foulhoux, L. and M.M. Bernitsas, (1993), "Forces and Moments on a Small Body Moving in a 3-D Unsteady Flow," Journal of Offshore Mechanics \& Arctic Engineering, ASME Transactions, Vol.115, No.2, May, pp. 91-104.

Gabbai, R. D., Benaroya, H. 2005. An overview of modeling and experiments of vortexinduced vibration of circular cylinders. Journal of Sound and Vibration 282, 575-616.

Gartshore, I. S., Khanna, J., Laccinole, S. 1978. The effectiveness of vortex spoilers on a circular cylinder in smooth and turbulent flow. Proceeding $5^{\text {th }}$ International Conference on Wind Engineering Fort Collins, CO.

Gerrard, J. H. 1966. The mechanics of the formation region of vortices behind bluff bodies. Journal of FluidMechanics 25, 401-413.

Govardhan, R., Williamson, C. H. K. 2000. Modes of vortex formation and frequency response of a freely vibrating cylinder. Journal of FluidMechanics420, 85-130.

Govardhan, R., Williamson, C. H. K. 2006. Defining the modified Griffin plot in vortexinduced vibration: revealing the effect of Reynolds number using controlled. Journal of FluidMechanics 561, 147-180.

Gowda, B.H.L., Kumar, A.R. 2006. Flow-induced oscillations of a square cylinder due to interference effects. Journal of Sound and Vibration 297, 842-864.

Güven, O., Farell, C., Patel, V.C. 1980. Surface-roughness effects on the mean flow past circular cylinders. Journal of FluidMechanics 98, 673-701. 
Higuchi, H., Kim, H. J., Farell, C. 1989. On flow separation and reattachment around a circular cylinder at critical Reynolds numbers. Journal of FluidMechanics 200,149-171.

Honji, H., Taneda, S. 1969. Time dependent flow around a circular cylinder accelerated uniformly from one speed to another. Report of the Research Institute of Applied Mechanics (Kyushu University, Japan) 17, 187-193.

Khalak, A., Williamson, C. H. K. 1996. Dynamics of a hydroelastic cylinder with very low mass and damping. Journal of Fluids and Structures 10, 455-472.

Khalak, A.,Williamson, C. H. K. 1999. Motions, forces and mode transitions in vortexinduced vibrations at low mass-damping. Journal of Fluids and Structures 13, 813-851.

Korkischko, I., Meneghini, J. R. 2010. Experimental investigation of flow-induced vibration on isolated and tandem circular cylinders fitted with strakes. Journal of Fluids and Structures 26, 611-625.

Krishnamoorthy, S., Price, S.J., and Paidoussis, M.P. 2001. Cross flow past an oscillating circular cylinder: synchronization phenomenon in the near wake. Journal of Fluids and Structure 15, 955-980.

Kumar, A. R., Sohn, C. H., Gowda, B. H. L. 2008. Passive control of vortex-induced vibrations: An overview. Recent Patents on Mechanical Engineering1, 1-11.

Kumar, A. R., Sohn, C. H., Gowda, B. H. L. 2009. Influence of corner radius on the near wake structure of a transversely oscillating square cylinder. Journal of Mechanical Science of Technology 23-9, 2390-2416.

Kumar, A.R., Gowda, B.H.L. 2006. Flow-induced vibration of a square cylinder without and with interference. Journal of Fluids and Structures 22, 345-369.

Lee, S. J.,Kim, H. B. 1997. The effect of surface protrusions on the near wake of a circular cylinder. J. of Wind Engineeringand Industrial Aerodynamics 69-71, 351-361.

Lubbad, R. K., Loset, S., Gudmestad, O. T.,Torum, A., Moe, G. 2007. Vortex induced vibrations of slender marine risers-effects of rounded-sectioned helical strakes. Proceedings 17th ISOPE Conference, Lisbon, Portugal, July 1-6.

Luo, S.C. 1992. Vortex wake of a transversely oscillating square cylinder: A flow visualization analysis. Journal of Wind Engineering and Industrial Aerodynamics 45-1, 97-119.

Morse, T.L., Williamson, C.H.K. 2009. Prediction of vortex-induced vibration response by employing controlled motion. Journal of Fluids Mechanics 634, 5-39. 
Muddada, S., Patnaik, B. S. V. 2010. An active flow control strategy for the suppression of vortex structures behind a circular cylinder. European Journal of Mechanics-B/Fluids 29, 93-104.

Nakamura, Y., Tomonari, Y. 1982. The effects of surface roughness on the flow past circular cylinder at high Reynolds numbers. Journal of Fluid Mechanics 123, 363-378.

Nebres, J., Batill, S. 1993. Flow about a circular cylinder with a single large-scale surface perturbation. Experiments in fluids 15, 369-379.

Ongoren, A., Rockwell, D. 1988. Flow structure from an oscillating cylinder Part 1: Mechanisms of phase shift and recovery in the near wake. Journal of Fluid Mechanics 191, 197-223.

Païdoussis, M. 1983. A review of flow-induced vibrations in reactors and reator components. Nuclear Engineering and Design 74-1, 31-60.

Park, H., Bernitsas, M. M.,Kumar, A. R. 2011. Using the map of passive turbulence control to flow induced motions to suppress circular cylinder motion at $31,000<\operatorname{Re}<120,000$. Proceedings OMAE 2011, Rotterdam, The Netherlands, June 1924, 2011; also, ASME Journal of Offshore Mechanics and Arctic Engineering ASME Trans., Vol.134, No. 4, Nov. 2012.

Park, H., Kumar, A. R., Bernitsas, M. M. 2013a. Enhancement of flow-induced motion of rigid circular cylinder on springs by localized surface roughness at $3 \times 10^{4} \leq \mathrm{Re} \leq 1.2 \times 10^{5}$ Ocean Engineering, in revision 2013.

Park, H., Bernitsas, M. M. Chang, C. C. 2013b. Map of passive turbulence controls to flow-induced motions for a circular cylinder at 30,000 $<\operatorname{Re}<120,000$ : Sensitivity to zone convering. Proceedings OMAE 2013, Nantes, France, June 9 -14, 2013.

Raghavan, K.,Bernitsas, M. M. 2011. Experimental investigation of Reynolds number effect on vortex induced vibration of rigid cylinder on elastic supports. Ocean Engineering, Vol.38, \#5-6, pp.719-731.

Sarpkaya, T. 1979. Vortex-induced oscillations - a selective review. Journal of Applied Mechanics 46, 241-258.

Sarpkaya, T. 2004. A critical review of intrinsic nature of vortex-induced vibrations. Journal of Fluids and Structures 19, 389-447.

Schaudt, K. J., Wajnikonis, C., Spencer, D., Xu, J., Leverette, S., Masters, R. 2008. Bench marking of VIV suppression systems. Proceedings of the 27thConference on Ocean, Offshore, and Artic Engineering, Estoril, Portugal, 2008. 
Tombazis, N., Bearman, P. W. 1997. A study of three-dimensional aspects of vortex shedding from a bluff body with a mild geometric disturbance. Journal of Fluid Mechanics 330, 85-112.

Triantafyllou, M. S., Hover, F. S., Techet, A. H. 2004. The effect of Reynolds number on VIV: from subcritical to supercritical flow. Proc. 8th Intl Conf. on Flow Induced Vibrations, FIV 2004, Paris, France, 6-9 July (ed. E. de Langre., and F. Axisa), p. 303.

Tritton, D.J. 1977. Physical fluid dynamics, Van Nostrand Reinhold.

Vikestad, K., Vandiver, J. K., Larsen, C. M. 2000. Added mass and oscillation frequency for a circular cylinder subjected to vortex-induced vibrations and external disturbance. Journal of Fluids and Structures 14, 1071-1088.

Wakahara, T., Ohyama, T., Fujii, K. 1992. Suppression of wind-induced vibration of a tall building using tuned liquid damper. Journal of Wind Engineering and Industrial Aerodynamics 43 (1-3), 1895-1906.

Walker, D. T., Lyzenga, D. R., Ericson, E. A., Lund, D. E. 1996. Radar backscatter and surface roughness measurements for stationary breaking waves. Proceedings of the Royal Society Lond. A 452-1952, 1953-1984.

Williamson, C. H. K. 1996. Vortex dynamics in the cylinder wake. Annual Review of Fluid Mechanics 28, 477-539.

Williamson, C. H. K., Govardhan, G. 2004. Vortex-induced vibrations. Annual Review of Fluid Mechanics 36, 413-455.

Williamson, C. H. K., Roshko, A. 1988. Vortex formation in the wake of an oscillating cylinder. Journal of Fluids and Structures 2, 355-381.

Wong, H. Y., Kokkalis, A. 1982. A comparative study of three aerodynamic devices for suppressing vortex-induced oscillation. Journal of Wind Engineeringand Industrial Aerodynamics 10, 21-29.

Zdravkovich, M. M. 1981. Review and classification of various aerodynamic and hydrodynamic means for suppressing vortex shedding. Journal of Wind Engineering and Industrial Aerodynamics 7, 145-189.

Zdravkovich, M. M. 1997. Flow around circular cylinders Volume 1: Fundamentals. Oxford University Press, England. 


\section{List of Tables}

Table 1.Oscillator particulars 


\section{List of Figures}

Fig. 1. Map of FIM induced by selectively located roughness: (a) Sandpaper P180, (b) Sandpaper P60.

Fig. 2. PTC location and coverage on the cylinder surface.

Fig. 3. Amplitude and frequency response for smooth cylinder and weakly suppressed PTC-cylinder

Fig. 4. Displacement time histories for smooth circular cylinder

Fig. 5. Wake vortex structure of smooth cylinder over a cycle: $U^{*}=5.58$ (upper end of initial branch), $\operatorname{Re}=4.33 \times 10^{4}, A^{*}=0.404, f_{\text {osc }} / f_{s}=0.64$.

Fig. 6. Wake vortex structure of smooth cylinder over a cycle: $U^{*}=8.18$ (upper branch), $\operatorname{Re}=6.35 \times 10^{4}, A^{*}=1.39 ; 2 \mathrm{P}$ pattern. Occasionally, a 3P pattern is observed at the same $U^{*}$. Picture (k) shows 4 of the 6 vortices in the 3P pattern.

Fig. 7. Wake vortex structure of smooth cylinder in lower branch, $U^{*}=12.28$, $\operatorname{Re}=9.53 \times 10^{4}, A^{*}=0.76$. Sketches (a)-(f) show the cycle with seven shed vortices. Variations exist. Sketch (g) is a variation of (b) and sketch (h) is a variation of (d).

Fig. 8. Typical wake vortex structure of smooth cylinder at $U^{*}=14.51$ (desynchronization), $\operatorname{Re}=1.12 \times 10^{5}, A *=0.06$;

Fig. 9. WS1 displacement time histories and spectra: (a)-(d) P180:0 $0^{\circ}-8^{\circ}$

Fig. 10. Dye visualization of flow reattachment in the WS1 zone at $U^{*}=5.21$.

Fig. 11. Vortex formation length for smooth cylinder and PTC-cylinder at $U^{*}=5.21$ : (a) smooth cylinder, (b) WS1, (c) WS2.

Fig. 12.Amplitude and frequency response in the strong suppression zone

Fig. 13. SS displacement time histories: (a)-(d) P180:70 $-86^{\circ}$ (e) P180:90 $90^{\circ}-106^{\circ}$

Fig. 14. Vortex formation length for smooth cylinder and PTC-cylinder at $U^{*}=5.58$ : (a) smooth cylinder, (b) SS (P180: $\left.70^{\circ}-86^{\circ}\right)$ 


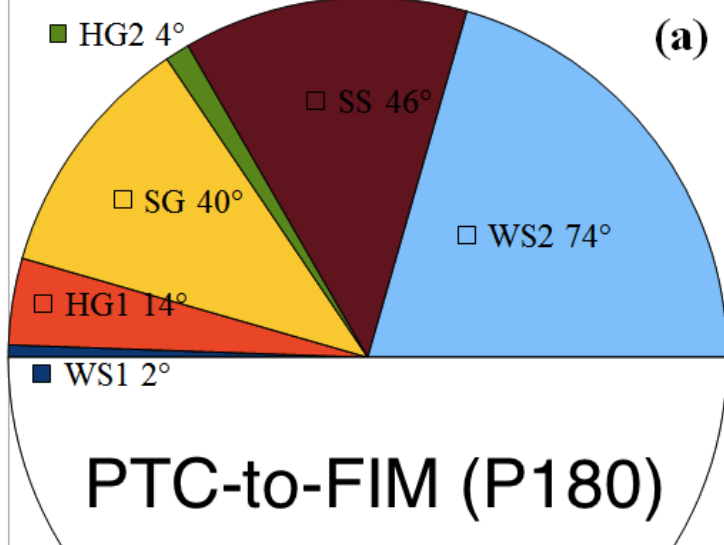

WS1 (Weak suppression1)

HG1 (Hard Galloping 1)

SG (Soft Galloping)

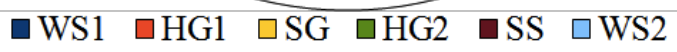

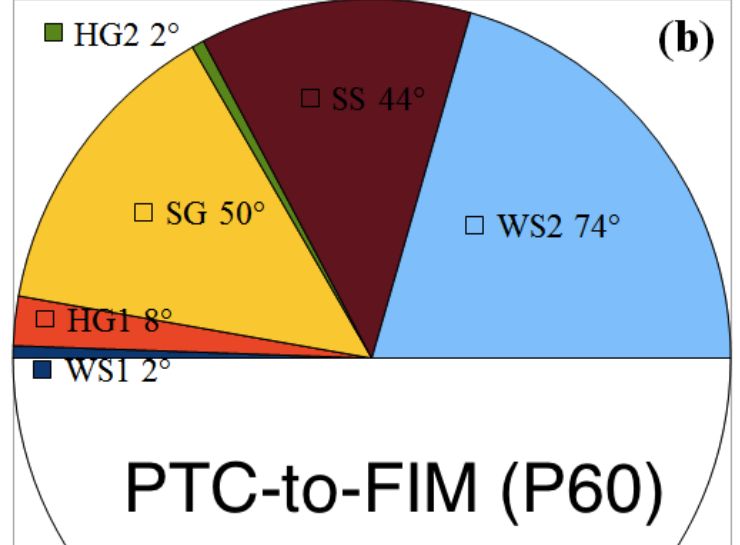

HG2 (Hard Galloping 2)

SS (Strong Suppression)

WG2 (Weak Suppression 2)

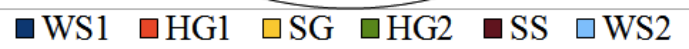


(a)

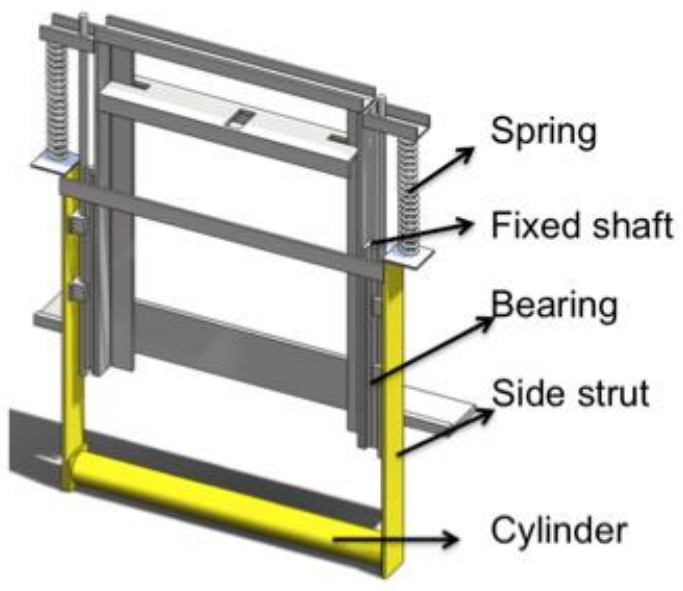

PTC

(b) Placement (Roughness) Coverage Area \begin{tabular}{l|l} 
angle $\left(a_{\text {PTC }}\right)$ & $\begin{array}{l}\text { Coverage Area } \\
\text { (width of strip) }\end{array}$
\end{tabular}

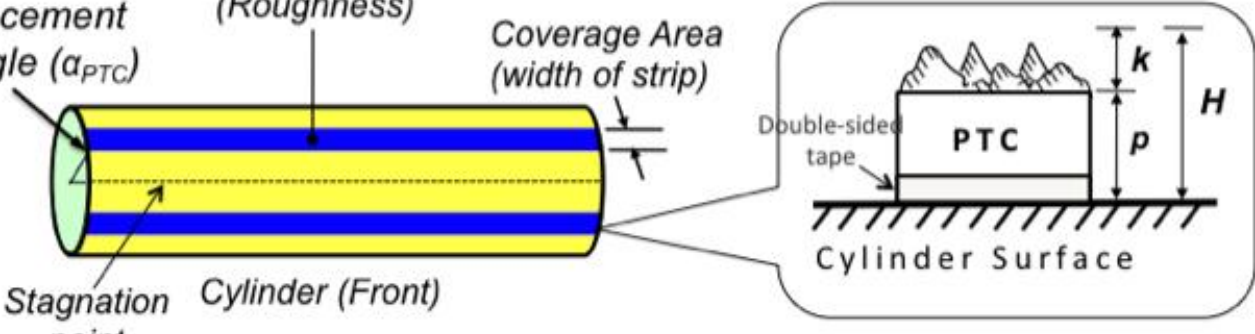

point 

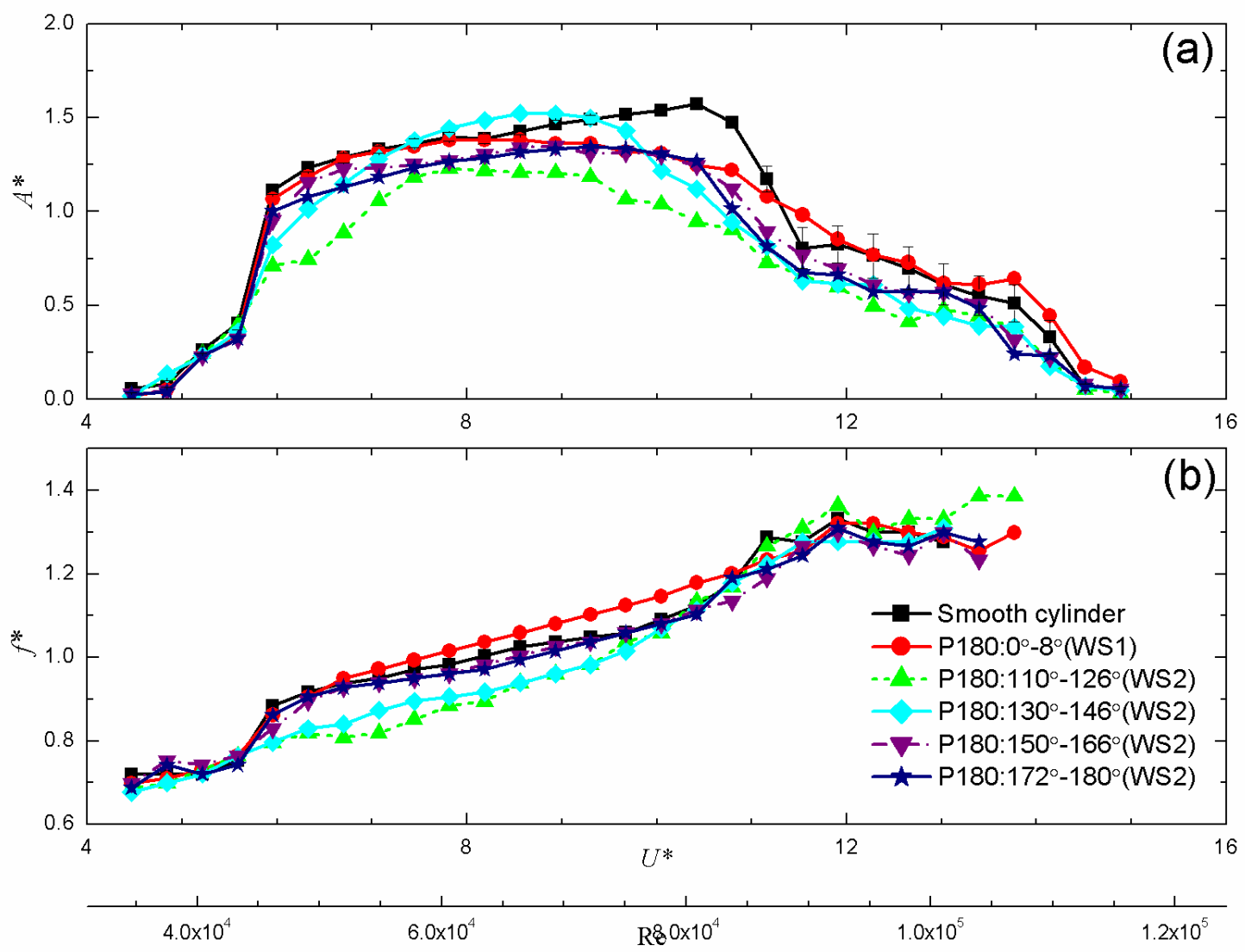

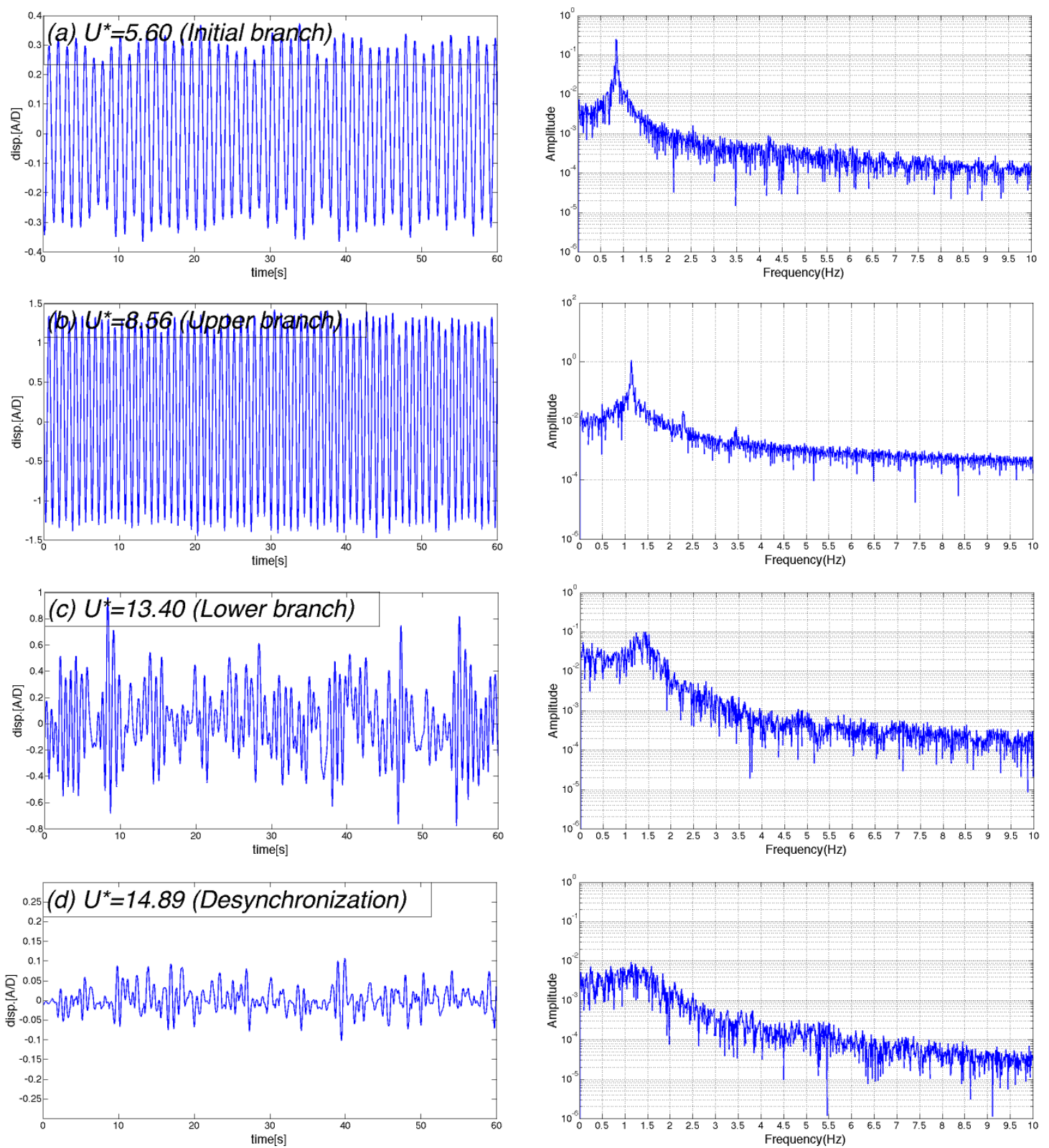

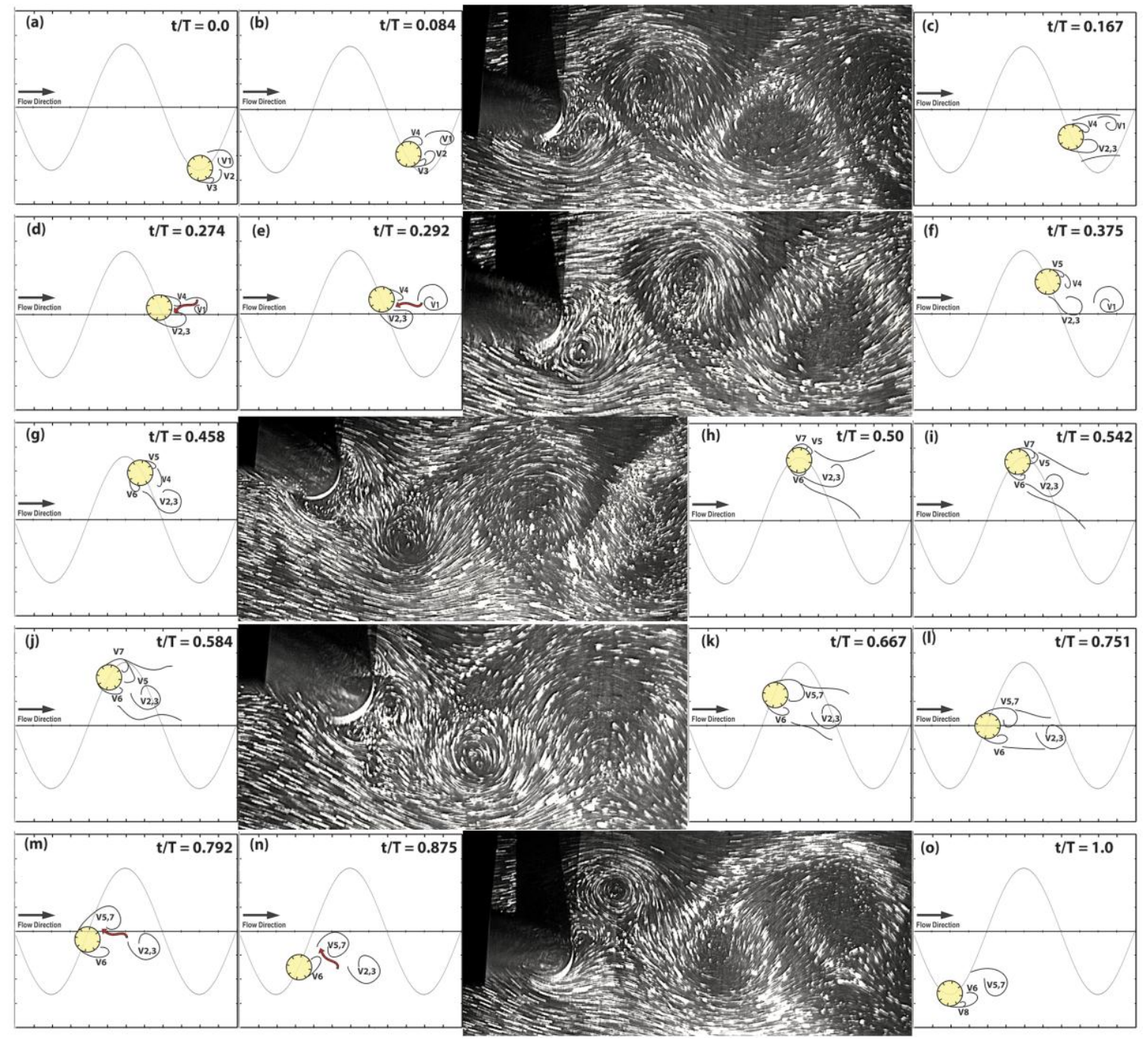

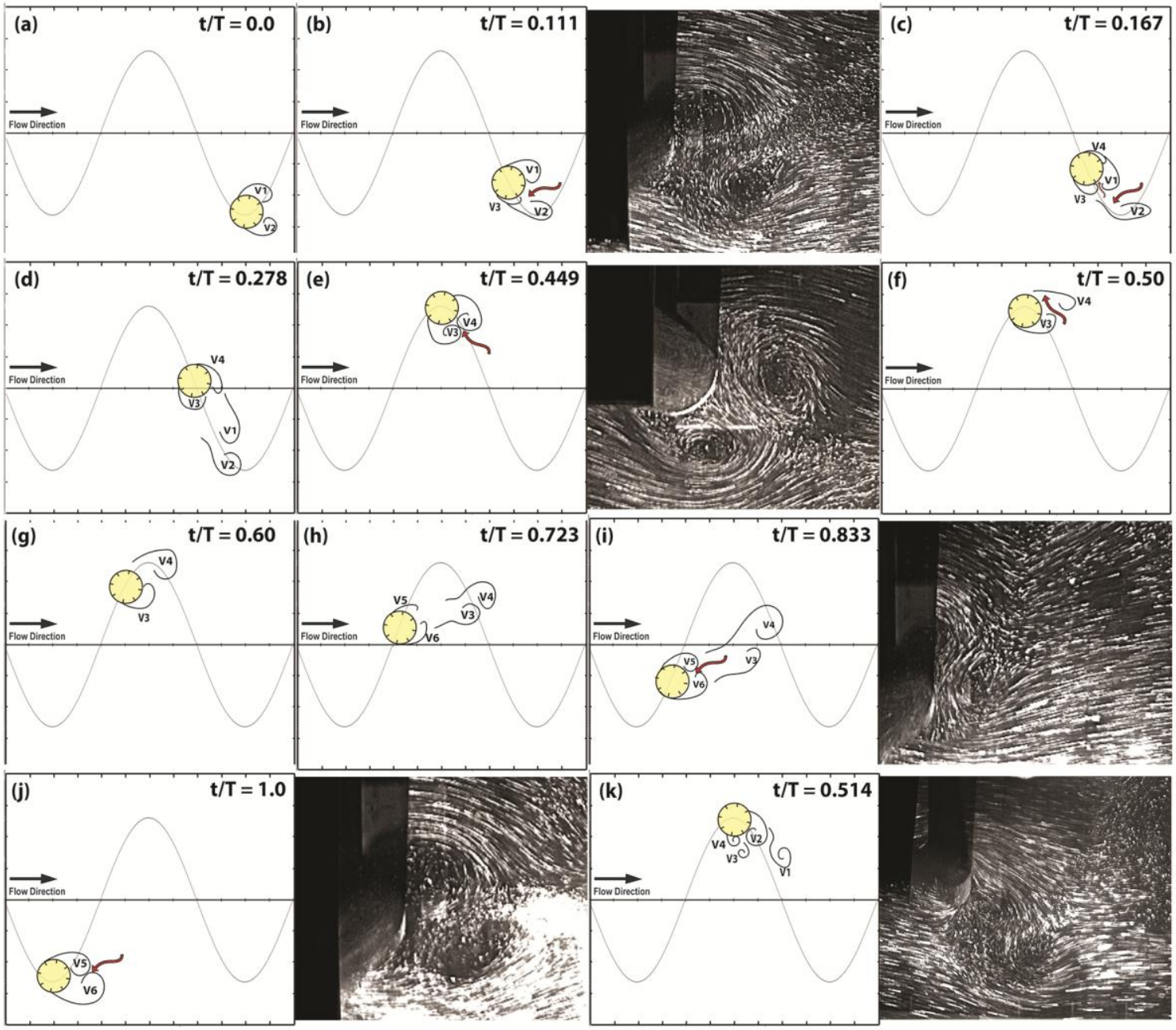


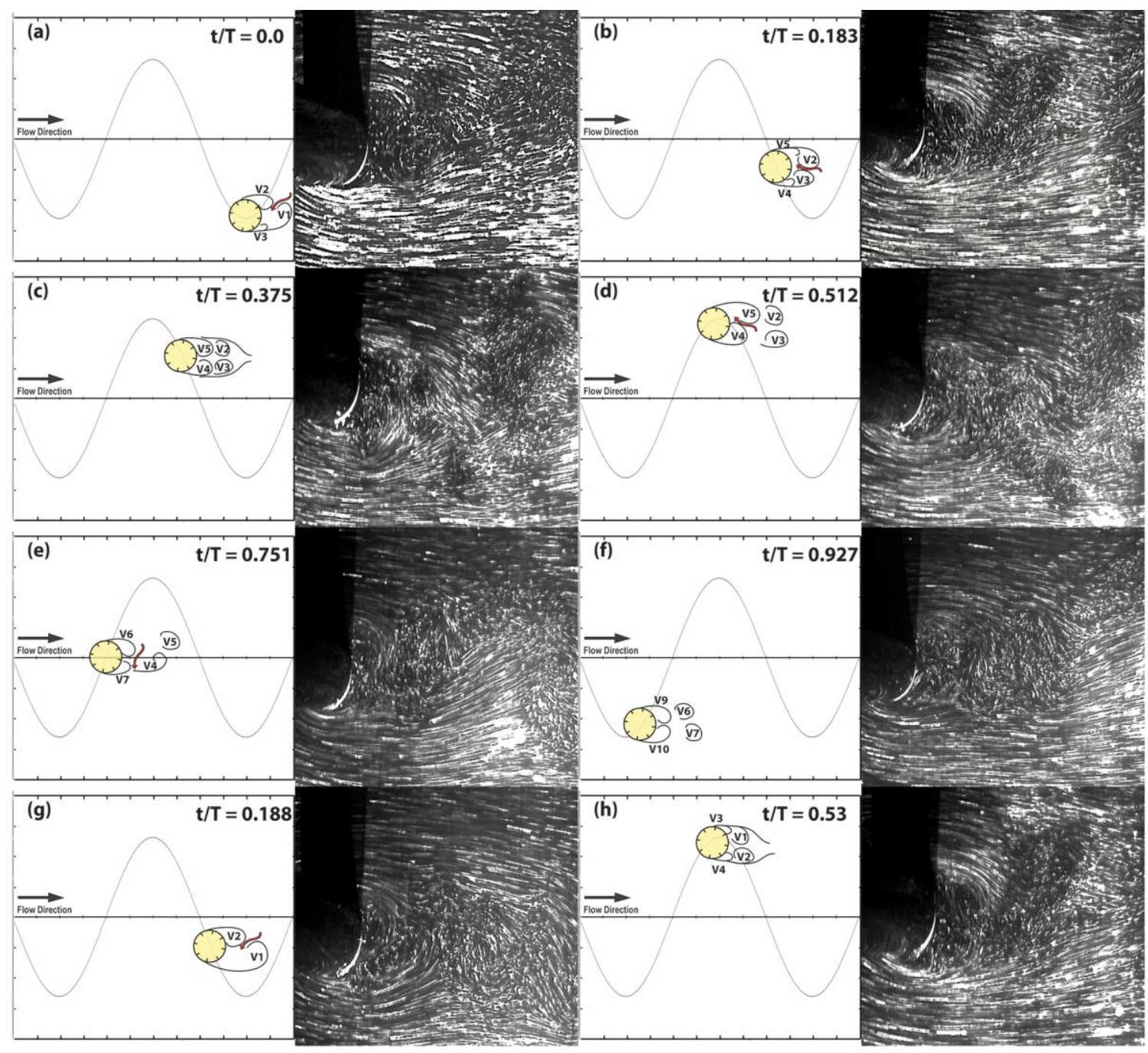



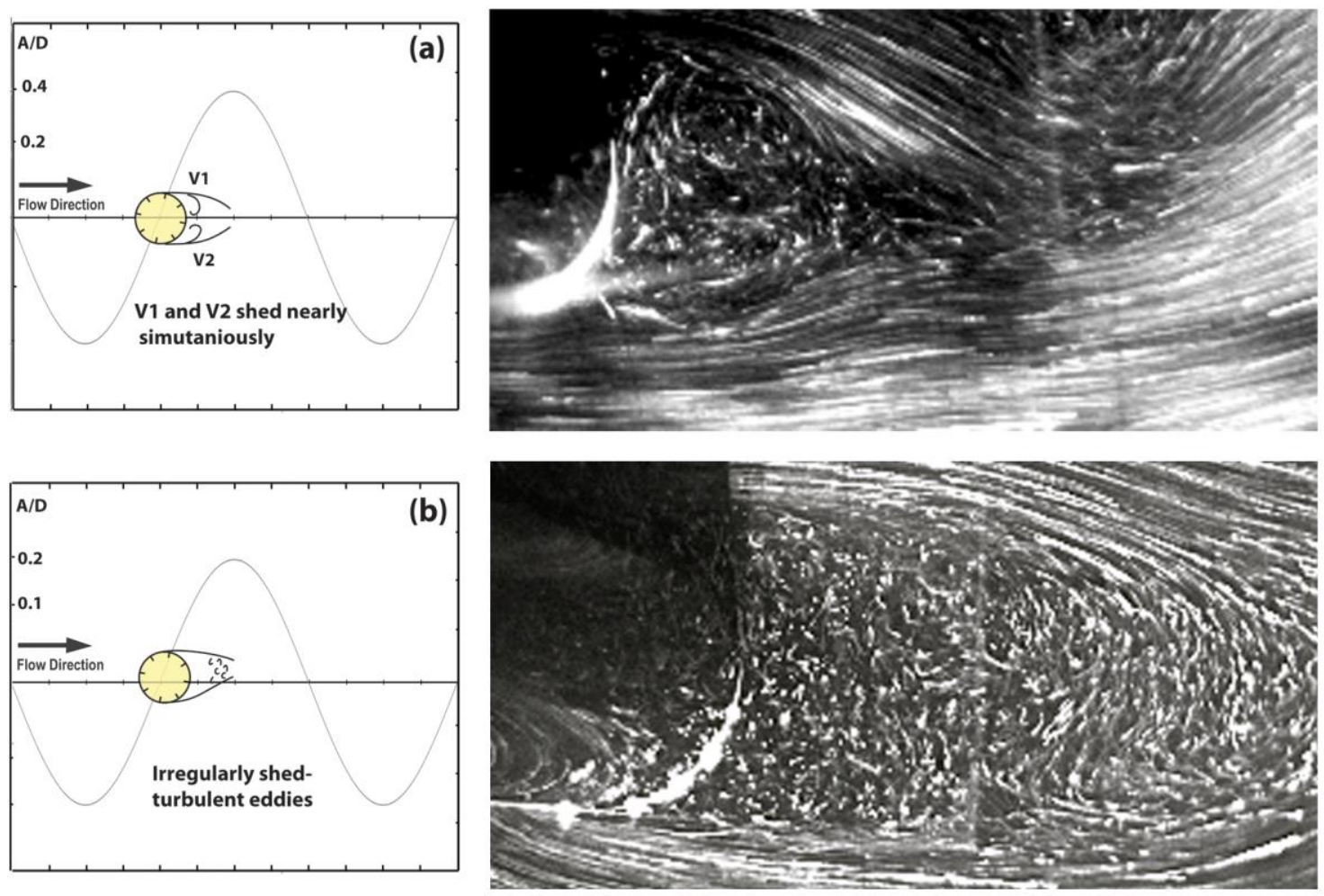

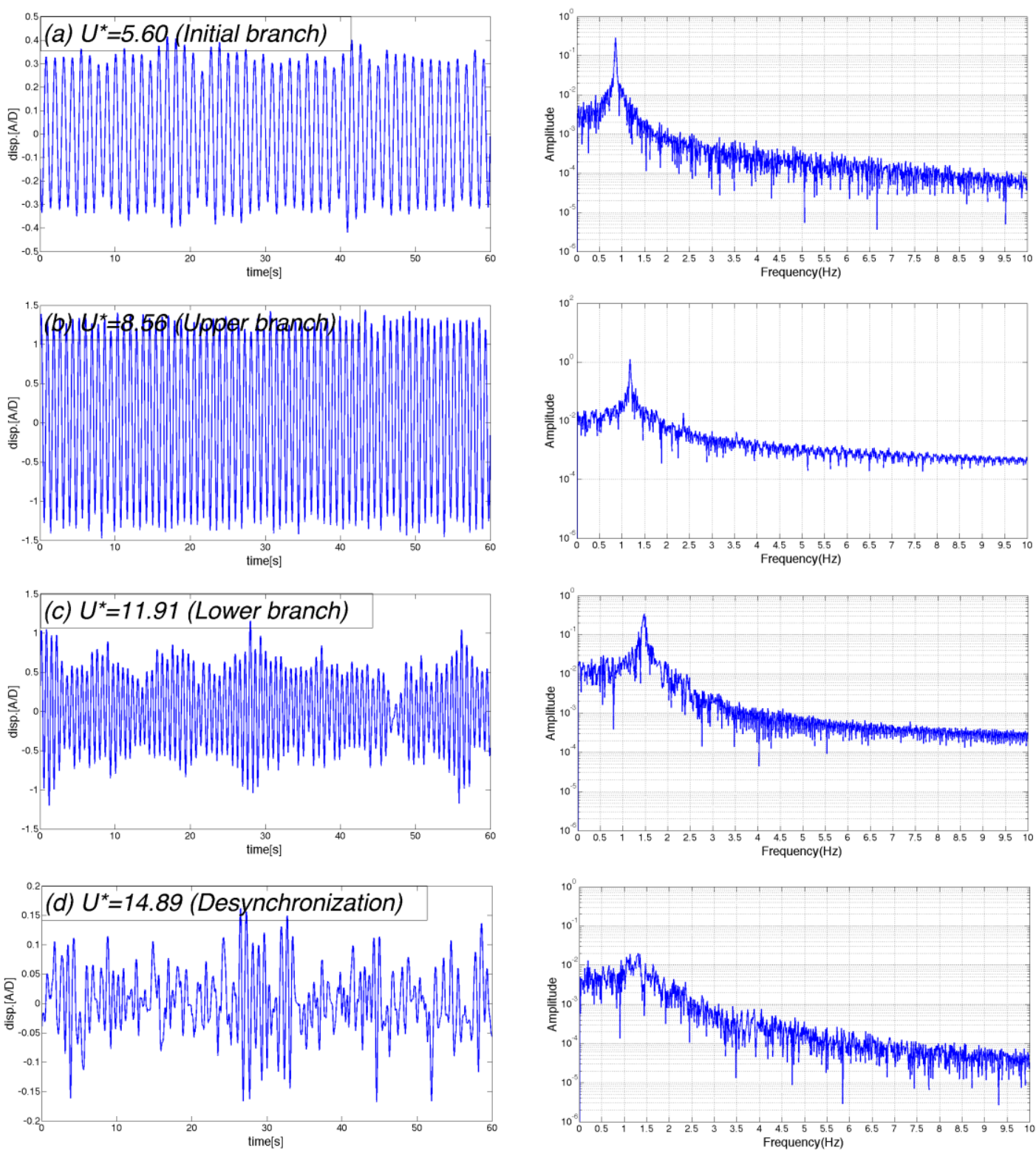


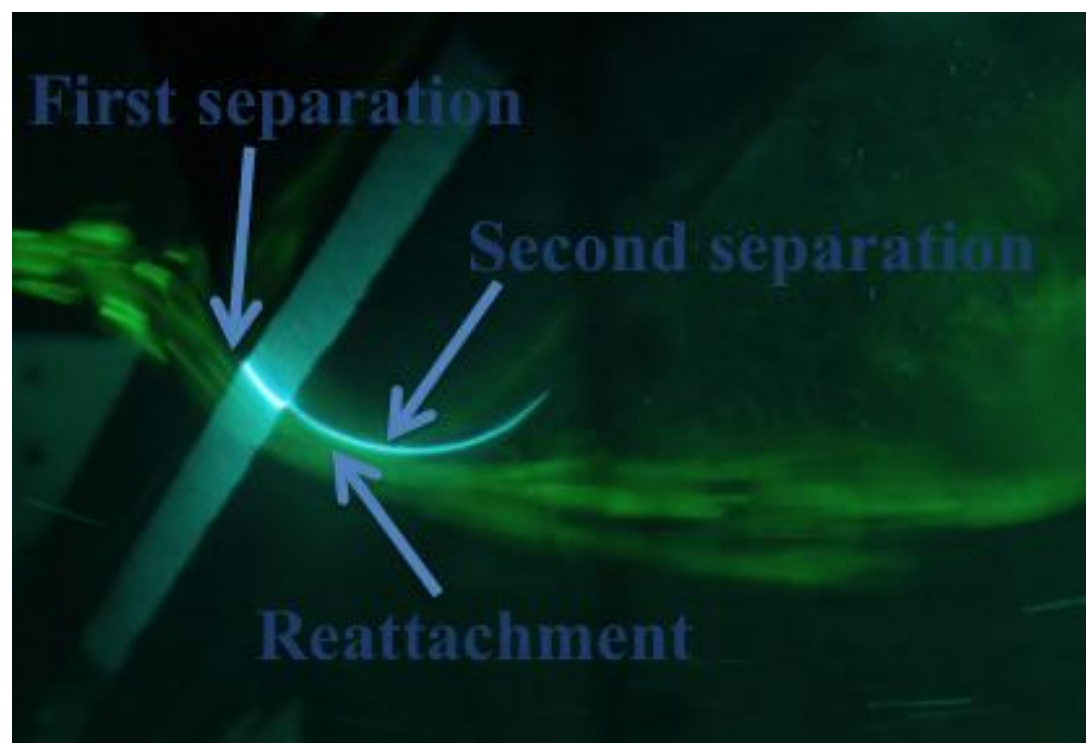



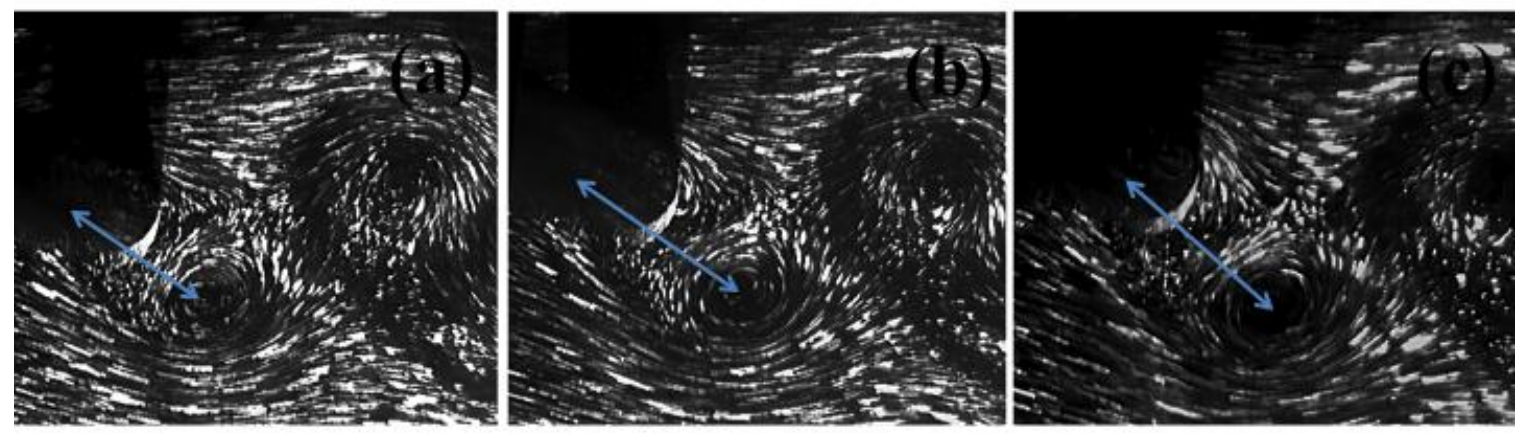

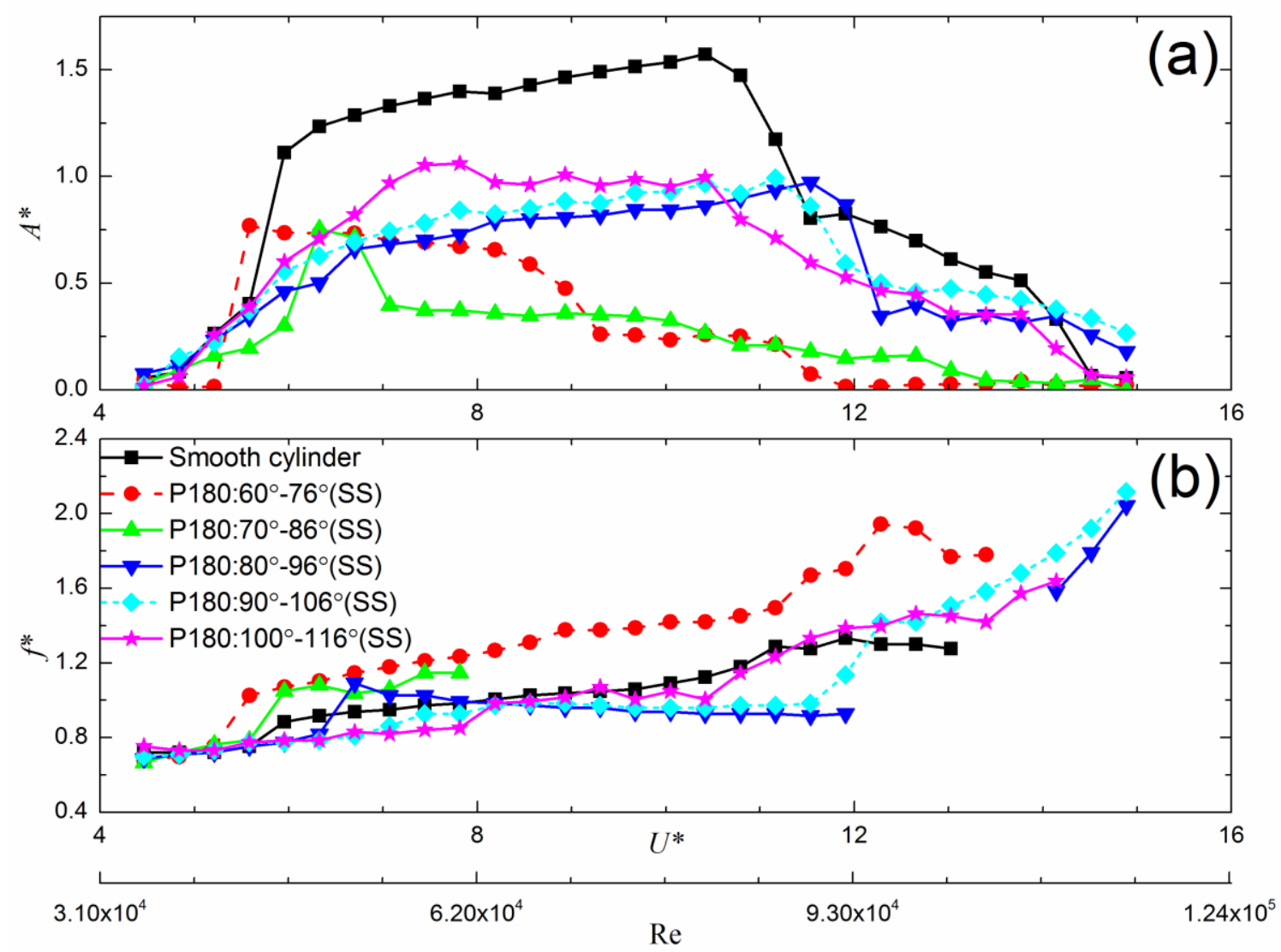

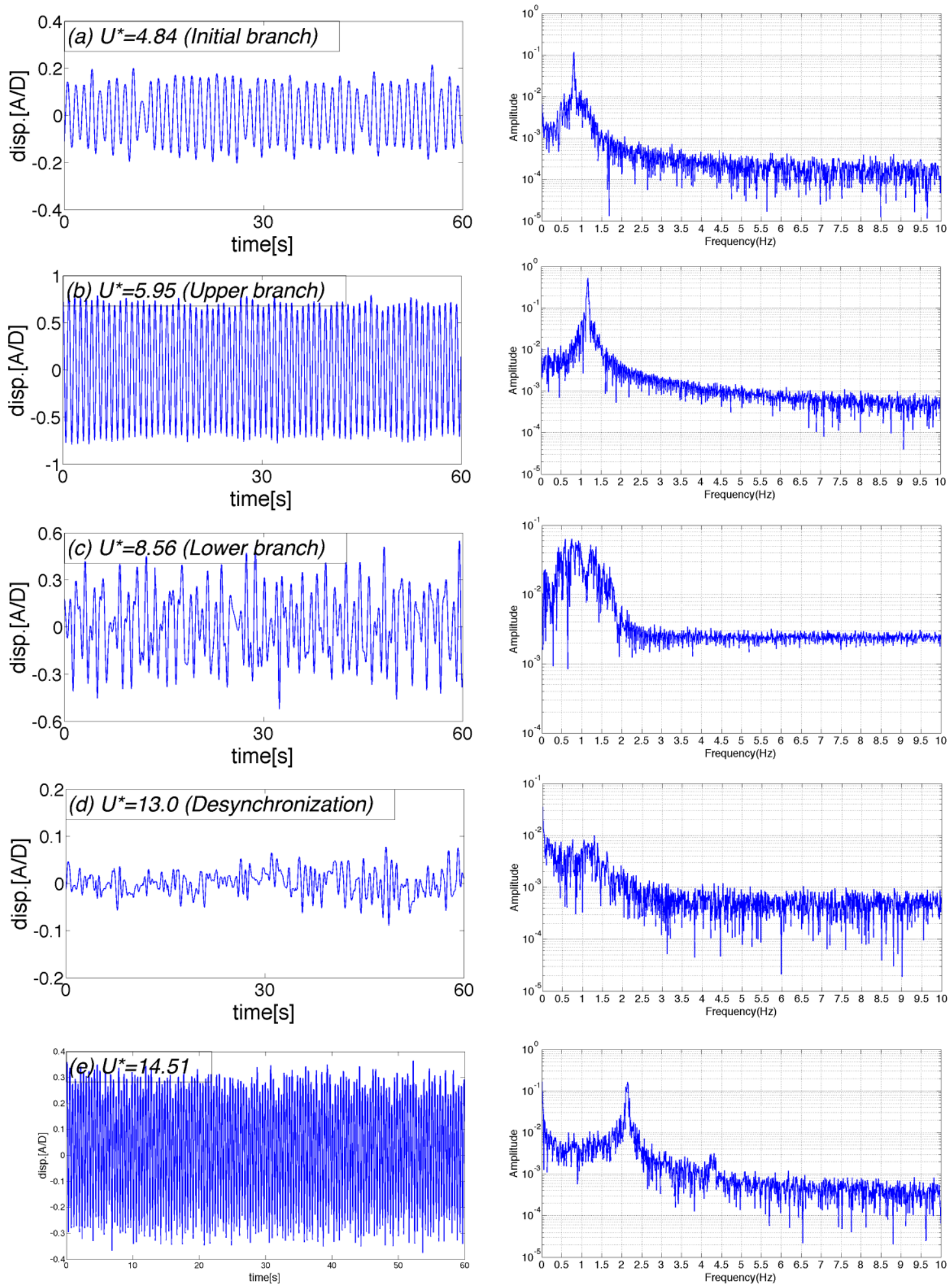

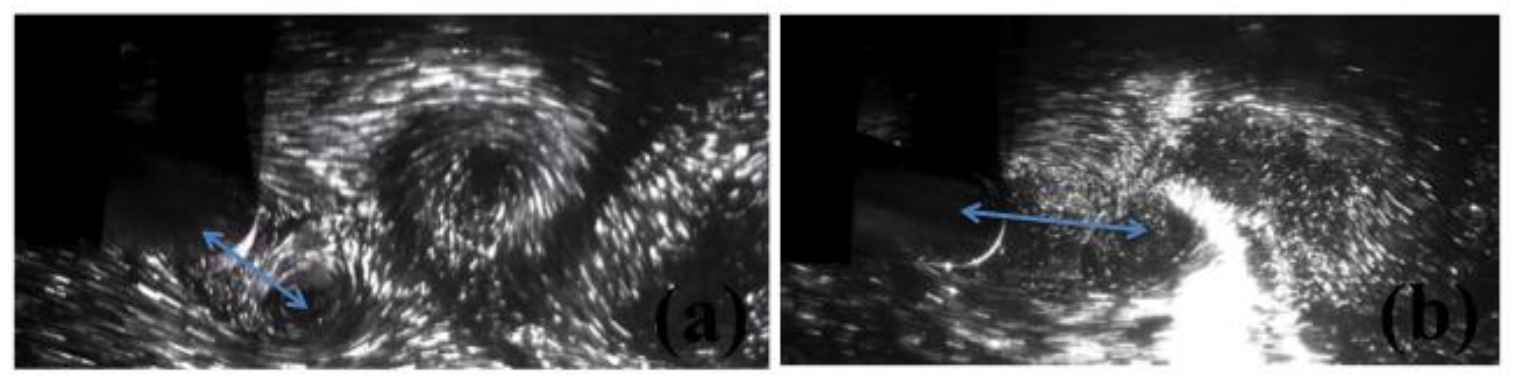
Table 1. Oscillator particulars

\begin{tabular}{ll}
\hline$D=$ cylinder diameter & $8.89 \mathrm{~cm}$ \\
$K=$ spring constant & $763 \pm 2.4 \mathrm{~N} / \mathrm{m}$ \\
$L=$ cylinder length & $91.44 \mathrm{~cm}$ \\
$m_{\text {cylinder }}=$ mass of the bare cylinder & $2.535 \mathrm{Kg}$ \\
$m_{o s c}=$ total oscillating mass including $1 / 3^{\text {rd }}$ & $9.784 \mathrm{Kg}$ \\
of the spring mass & \\
$m_{d}=$ displaced fluid mass & $5.6707 \mathrm{Kg}$ \\
$m_{A}=$ added mass & $5.6707 \mathrm{Kg}$ \\
$m^{*}=$ mass ratio & 1.725 \\
$f_{n, w}=$ natural frequency in still water & $1.118 \mathrm{~Hz}$ \\
$\zeta=$ damping ratio & $0.0158 \pm 0.0008$ \\
\hline
\end{tabular}

